\title{
On the Tineidae of the Southern Arabian Peninsula and Sudan (Lepidoptera: Tineidae)
}

\author{
With 75 figures and 1 table
}

REINHARD GAEDIKE ${ }^{1}$

${ }^{1}$ Florusstraße 5, 53225 Bonn, Germany. - tinagma@msn.com

Published on 2014-12-15

\section{Summary}

Twenty species new to science are described and illustrated: Hapsifera gozmanyi, Hapsiferona jemenitica, H. remanei, Psecadioides stuebneri, Heterostasis hackeri, Pachyarthra africana, Edosa pareffulgens, E. albicapitella, E. furcata, Hyperbola lehmanni, H. sexspinosa, Perissomastix (Perissomastix) jemenitica, Trichophaga atripunctella, Proterospastis cornuta, P. spinosa, Crypsithyris luteocapitata, C. unipuncta, Ceratobia sudanica, Amphixystis minuta, A.taurus. The taxon hemiphracta Meyrick, 1926, hitherto regarded as a member of the genus Crypsithyris, is transferred to Ceratobia. New country records are established for ten species from Oman, 19 species from Yemen, and three species from the Sudan. The hitherto unknown females of Perissomastix (Perissomastix) wadimaidaq Gaedike, 2009 and P. (P.) taeniaecornis (WALsInghaM, 1896) are described. In the studied area 67 species are now known. The tineid fauna of Yemen comprises a mixture of Palaearctic and Afrotropical elements.

\section{Key words}

Lepidoptera, Tineidae, Arabian Peninsula, Sudan, original descriptions, faunistics

\section{Zusammenfassung}

Es werden 20 Arten als neu festgestellt, beschrieben und illustriert: Hapsifera gozmanyi, Hapsiferona jemenitica, H. remanei, Psecadioides stuebneri, Heterostasis hackeri, Pachyarthra africana, Edosa pareffulgens, E. albicapitella, E. furcata, Hyperbola lehmanni, H. sexspinosa, Perissomastix (Perissomastix) jemenitica, Trichophaga atripunctella, Proterospastis cornuta, P. spinosa, Crypsithyris luteocapitata, C. unipuncta, Ceratobia sudanica, Amphixystis minuta, A. taurus. Die bisher in der Gattung Crypsithyris stehende Art hemiphracta MeYrick, 1926 wird in die Gattung Ceratobia versetzt. Für den Oman konnten zehn Arten, für den Jemen 19 Arten und für den Sudan drei Arten als Erstfunde registriert werden. Von Perissomastix (Perissomastix) wadimaidaq GAEDIKe, 2009 und Perissomastix (Perissomastix) taeniaecornis (WALsInGHAM, 1896) werden die bisher unbekannten Weibchen beschrieben. Im untersuchten Gebiet sind bisher 67 Arten nachgewiesen. Die Artenzusammensetzung im Jemen ist eine Mischung aus paläarktischen und afrotropischen Elementen.

\section{Introduction}

During recent years I have had the opportunity to study material from a faunistically very interesting area of the Palaearctic. The specimens were collected during

several collecting trips to Yemen and to Oman. Additionally, some specimens from Sudan were included in the investigation. 
The material was collected by the late M. Fibiger and colleagues, now in the collection of the Zoological Museum, Copenhagen, Denmark; by H. Hacker and colleagues, now in the collection of the Museum für Naturkunde der Humboldt-Universität, Berlin, Germany; by R. Remane, now in the collection of the Zoologische Staatssammlung, München, Germany; by R. Linnavuori, now in the collection of the Finnish Museum of Natural History, Helsinki, Finland; by the late L. Lehmann, now in the collection of A. Stübner, and some specimens from the collection of Georg Derra, Reckendorf, Germany.

The study shows that especially the fauna of the Yemen contains a mixture of Palaearctic and Afrotropical elements. More detailed studies of the fauna of the Sudan could reveal to what extent Palaearctic elements are present in the Afrotropical region.

For the possibility to study this material I thank G. Derra, Reckendorf, L. Kaila (FMNH, Helsinki), O. Karsholt (ZMUC, Copenhagen), W. Mey (ZMHB, Berlin), A. Segerer (ZSM, Munich), A. Stübner, Peitz.

I thank Andrew Liston (Müncheberg) for linguistic corrections and Ch. Kutzscher (SDEI, Müncheberg) for preparing the colour photographs.

\section{Abbreviations}

coll. Derra Georg Derra, Reckendorf, Germany

coll. Stübner Andreas Stübner, Peitz, Germany

FMNH Finnish Museum of Natural History, Helsinki, Finland

SDEI Senkenberg, Deutsches Entomologisches Institut, Müncheberg, Germany

SMNK Staatliches Museum für Naturkunde, Karlsruhe, Germany

ZMHB Museum für Naturkunde, Leibniz Institut für Evolutions- und Biodiversitätsforschung, Berlin, Germany

ZMUC Zoological Museum, Copenhagen, Denmark

ZSM Zoologische Staatssammlung, München, Germany

\section{Systematics}

Hapsiferinae Zagulajev, 1968

Hapsifera luridella ZelLeR, 1847

Saudi Arabia: Petersen, 1961b; Petersen \& Gaedike, 1982.

Bahrain Islands: Petersen, 1964.

Oman: $10^{\top}$, Prov. Dhofar, $19 \mathrm{~km}$ W Al-Mughsayl, $790 \mathrm{~m}, 16^{\circ} 50^{\prime} 27,4^{\prime \prime} \mathrm{N}, 53^{\circ} 40^{\prime} 78,5^{\prime \prime} \mathrm{E}, 23 .-24 . x i i .2009$, leg. L. Lehmann et al.; coll. Stübner:

First country record.

Yemen: Petersen, 1961a.
New records: $20^{\star}$, Prov. Sana'a, $15^{\circ} 18^{\prime} 12^{\prime \prime N}, 44^{\circ} 16^{\prime} 48^{\prime \prime} E$, mountains sw Sana'a, Jabal' Ayban, Bait N’arna, 2700 m, 18.iv.1998, leg. M. Fibiger et al., ZMUC; coll. Derra.

The species is wide distributed from North Africa through the Near and Middle East.

\section{Hapsifera punctata PETERSEN, 1961}

Saudi Arabia: Petersen, 1961a; Petersen \& Gaedike, 1982.

\section{Hapsifera gozmanyi sp. n.}

Holotype: $0^{\star}$, “Yemen, prov. Sana’a, $15^{\circ} 18^{\prime} 12^{\prime N}$, $44^{\circ} 16^{\prime} 48^{\prime \prime E}$, mountains SW Sana'a, Jabal 'Ayban, Bait Na’arna, 18.iv.1998, 2700 m, M. Fibiger leg.;" "Coll. ZMUC, Copenhagen Denmark;" "Gen.präp.[genitalia slide] Gaedike Nr. 6011;" "Holotypus ơ, Hapsifera gozmanyi sp. n., det. R. Gaedike 2013;" ZMUC; Paratypes: $1 \sigma^{\star}$, "Yemen, $600 \mathrm{~m}$, Prov. Al Hudaydah, Jabel, Burra, 25 km SE Bajil, 23.-24.iv.1998, leg. M. Fibiger et al.;" "Coll. ZMUC, Copenhagen Denmark;" "Gen.präp. [genitalia slide] Gaedike Nr. 6186;" ZMUC; 2 o", "[Genitalia slide Derra] 6405; 2nd specimen: 6406;" “Jemen, 18.iv.1998, 3I, Prov. Sana'a, 2700-2750 m, Jabal 'Ayban, Bait Na'arna, leg. Bischof, Bittermann, Fibiger, Hacker, Schreier;" Coll. Derra; SDEI.

Derivatio nominis: Named in honour of L. Gozmány, a specialist on the Afrotropical Tineidae.

Diagnosis (Figs 1-2): Wingspan 13-17 mm; head brush pale creamy, above palpi whitish; labial palpi inside pale creamy, second segment outside partly overlaid with brown scales, third segment thin, tapered; antennae dark creamy, scapus with pecten (antennae of holotype broken, one incomplete antenna glued); thorax and tegulae creamy, overlaid with brown scales, mostly on the first half; forewings creamy, with a pattern of dark brown patches basally, at dorsum at $1 / 3$, at costa on $1 / 2$, below cell at $2 / 3$ and subapically, some smaller patches at costa in the first half and below the patch at dorsum; the creamy scales with minute dark brown tip. Sometimes (fig. 2) the entire forewing overlaid with more brown colouration, the patches at costa and below cell connected; hindwing shiny light grey.

Male genitalia (Figs 25-26): Uncus truncated, with two pointed tips, gnathos arms thin, apically curved, with pointed tip, basally rounded; vinculum band-shaped, saccus visible only as minute tip; valvae as long as uncustegumen complex, parallel-sided, apically rounded; apodemes connected, bridge-shaped; anellus nearly triangular, fused with valvae through narrow bristled process, phallus about $2 / 3$ of the length of valva, subapically with indication of two teeth.

Female genitalia: Unknown. 
Remarks: Superficially similar to H. pachypsaltis GoZmány, 1965, but clearly smaller. In the male, genitalia differences are the thin gnathos arms, the minute saccus and phallus without clear teeth.

\section{Hapsifera rhodoptila MeYRICK, 1920}

Sudan: GoZMÁNY \& VÁRI, 1973.

Hitherto known from Kenya, Ethiopia and from Sudan.

\section{Hapsiferona glareosa (MEYRICK, 1912)}

Saudi Arabia: Petersen \& Gaedike, 1982.

Yemen: $1 \sigma^{\star}$, Ta’izz, Ta’izz, 22.-23.iv.1992, leg. R. Linnavuori, FMNH; $10^{\star}$, Prov. Ta'izz, Wadi Warazan, $5 \mathrm{~km} \mathrm{NW}$ Ar Rahidah, 1100 m, 26.iv.1998, leg. M. Fibiger et al.,

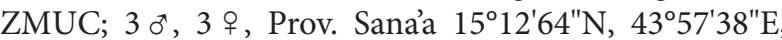
6 km NW Suq Baw’an, 3000 m, 20.iv.1998, leg. M. Fibiger,

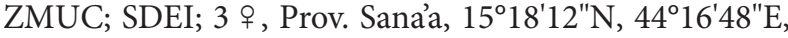
mountains sw Sana'a, Jabal 'Ayan, Bait Na'arna, 2700 m, 18.iv.1998, leg. M. Fibiger et al., ZMUC; coll. Derra; 1 ㅇ , Prov. Sana'a, 60 km SW Makaban Naqil Manakhah, 1730 m, 21.iv.1998, leg. M. Fibiger et al., ZMUC; 1 ơ $^{*}$, Prov. Al Hudaydah, Jabel, Burra, 25 km SE Bajil, 600 m, 23.-24.iv.1998, leg. M. Fibiger et al., SDEI:

First country records. Hitherto known from Zimbabwe and Tanzania (according to GozMÁNY \& VÁRI, 1973).

\section{Hapsiferona arabica GAEDIKE, 2009}

UAE: GAEDIKE, 2009.

Hitherto known only from the UAE.

\section{Hapsiferona jemenitica sp. n.}

Holotype: o", "Yemen, Prov. Abyan, $50 \mathrm{~km}$ NE Aden, $7 \mathrm{~km}$ NE Zinjibar, Wadi Bana, $50 \mathrm{~m}$, 29.iv.1998, leg. M. Fibiger et al.;" "Coll. ZMUC, Copenhagen Denmark;" "Gen.präp.[genitalia slide] Gaedike Nr.6012;" "Holotypus $\sigma^{\star}$, Hapsiferona jemenitica sp. n., det. R. Gaedike 2013;" ZMUC.

Derivatio nominis: Named after the country where the holotype was collected.

Diagnosis (Fig. 3): As the condition of the holotype is somewhat poor (head, thorax and wings partly rubbed), it is impossible to give a complete diagnosis. Wingspan $13 \mathrm{~mm}$; antennae as long as forewing, scapus with pecten; labial palpi whitish, second segment with long, apically dark scales, third segment short, thin, pointed; forewing creamy, with indications of dark brown pattern in the apical half; hindwings shiny grey.

Male genitalia (Figs 27-29): Uncus with two rounded edges, gnathos arms angled before $1 / 2$, narrowed to blunt apex; saccus narrow, as long as uncus-tegumen complex, valvae as long as saccus, parallel-sided, apically rounded only a little narrower as basally; phallus nearly twice longer as valva, basally rounded, thin, without any teeth.

Female genitalia: unknown.

Remarks: The very long thin phallus without any teeth make this species distinguishable from the other known members of this genus.

\section{Hapsiferona remanei sp. n.}

Holotype: o", "Sudan, El Damar, Hudeiba, 12.vi.1962, leg. R. Remane, Staats[samm]l[un]g München;" "Gen. präp.[genitalia slide] Gaedike Nr. 5556;" "Holotypus o", Hapsiferona remanei sp. n., det. R. Gaedike 2013;" ZSM; Paratype: $1 \sigma^{\star}$, "[Sudan] Hudeiba, 21.iii.1962, Lighttrap;" “Gen.präp.[genitalia slide] Gaedike Nr. 2483;” SDEI.

Derivatio nominis: Named after the collector, R. Remane.

Diagnosis (Fig. 4): Wingspan $16 \mathrm{~mm}$; head brush yellowish creamy; antennae a little longer than forewings, scapus white, with pecten, flagellum creamy; labial palpi pale creamy, scales subapically dark brown, second segment with long scales, third segment short, thin; thorax in the first quarter light brown, other parts creamy, tegulae brown, apically paler; forewing creamy, mixed with light and dark brown, without distinct pattern. Dark brown are a strip along base of fringe to apex with an oblique prolongation to a patch below costa at $3 / 4$, an indistinct marking at $1 / 2$ on cell and on base; hindwings shiny.

Male genitalia (Figs 30-32): Uncus with two rounded lobi, gnathos arms angled, basal half broader than apical half, tip rounded to pointed, area from uncus to the gnathos arms overlaid with a net of minute sclerotisations; saccus basally broad, narrower to blunt tip, as long as one gnathos arm; valvae as long as uncus-tegumen, basally broad, narrower to rounded apex, phallus as long as valva, more or less parallel-sided.

Female genitalia: unknown.

Remarks: Superficially distinguishable by the checkered pattern of forewings. In male, genitalia characterised by having short saccus.

\section{Scalidomia fetialis (MEYRICK, 1917)}

Yemen: 3 o, 2 ㅇ, Prov. Al Hudaybah, Jabel, Burra, $25 \mathrm{~km}$ SE Bajil, 23.-24.iv.1998, leg. M. Fibiger et al., ZMUC; SDEI; 1 ㅇ, Prov. Ta'izz, Wadi Warazan, 5 km NW Ar Rahidah, 1080 m, 27.iv.1998, leg. M. Fibiger et al., ZMUC; $2 \sigma^{*}$, Prov. Sana'a, 60 km SW Makaban, Naqil Manakhah, 1730 m, 21.iv.1998, leg. M. Fibiger et al., ZMUC; $2 \sigma^{\star}$, Prov. Ibb, 5 km NE Al Qa'jdah, 1850 m, 28.iv.1998, leg. M. Fibiger et al., ZMUC; SDEI: 
First records outside the African continent. For the records hitherto known from the African continent, see GOZMÁNY \& VARI (1973).

\section{Myrmecozelinae ZAGULAJEV, 1958}

\section{Heterostasis hackeri sp. n.}

Holotype: đđ, "Yemen, 3.xi.1996, 15.11/43.32, Bajil Khamis Bani Sa ad, 750 m, leg. Hacker;" “Gen.präp.[genitalia slide] Gaedike Nr. 5560;" "Holotypus ơ, Heterostasis hackeri sp. n., det. R. Gaedike 2013;" ZMHB.

Derivatio nominis: Named after the collector, H. Hacker.

Diagnosis (Fig. 7): Wingspan ca. $10 \mathrm{~mm}$; head with creamy adpressed scales, antennae with same colouration, thick, scapus without pecten; labial palpi inside creamy, outside somewhat darker; thorax, tegulae and forewings shiny golden, without any pattern; hindwings shiny whitish.

Male genitalia (Figs 36-38): Uncus with two pointed, bristled lobi, tegumen band-shaped, vinculum broad, with very long thin saccus; valvae with apodemes half of the length of phallus, apodemes nearly a half of the length of the entire valvae, inside the valvae an oblique, more strongly sclerotised, rod-shaped stripe with short thorns, costal edge concave, ventral edge in the first half parallel-sided, in the second half oblique to a rounded apex; phallus $2 / 3$ of the length of uncus-tegumen-saccus complex, narrow, subapically with minute strongly sclerotised tooth.

Female genitalia: Unknown.

Remarks: Superficially distinguishable from the only other member of this genus (H. extricata GozmánY, 1965) in lacking the violet sheen. In the male genitalia the shape of valva (concave costal edge, parallel-sided and then oblique ventral edge) and the minute subapical tooth on phallus are characteristic for the species.

The species is the first representative of this genus in the Palaearctic.

\section{Pachyarthra ochroplicella (CHRÉTIEN, 1915)}

Yemen: $1 \sigma^{\star}$, Prov. Shabwah, Wadi Hajar, 4 km S Hisn Bin Talib delta, 3.v.1998, leg. M. Fibiger et al., ZMUC; $10^{\text {*⿻ }}$, Prov. Ibb, 5 km non Al Qa'idah, 1840-1900 m, 28.iv.1998, leg. Bischof et al.; coll. Derra:

First country records. Hitherto known from Morocco, Algeria, Tunisia, Libya and Egypt.

\section{Pachyarthra iranica GAEDIKE, 2009}

UAE: GAEDIKE, 2009.

Oman: $4 \circ^{\star}, 1$ 우 , Northern region, $23^{\circ} 28^{\prime} \mathrm{N}, 58^{\circ} 14^{\prime} \mathrm{E}$, Al Hail, 7 km E Seeb, 23.xii.1992, 12., 14., 16.xii.1994, leg.
B. Skule, ZMUC; 1 o , Northern Region, Wadi Al Khawd, 100 m, 20 km S Seeb, 6.i.1993, leg. B. Skule, ZMUC; 1 o, 1 + Prov. Batinah, Al-Jabal al Ahdar Mts, Wadi Bani, $430 \mathrm{~m}, 23^{\circ} 20^{\prime} 0,13^{\prime \prime} \mathrm{N}, 57^{\circ} 29^{\circ} 23,5^{\prime \prime} \mathrm{E}, 19 .-20 . x i i .2009$, leg. L. Lehmann et al., coll. Stübner:

First country records. Hitherto known from the UAE and Iran.

\section{Pachyarthra grisea Petersen \& Gaedike, 1982}

Saudi Arabia: Petersen \& Gaedike, 1982.

UAE: GAEDIKE, 2009.

Yemen: 1 o , Prov. Abyan, $11 \mathrm{~km}$ SW Zinijbar, 4.v.1998, wadidunes, leg. M. Fibiger et al., ZMUC; 1 o, Prov. Hadramaul, $25 \mathrm{~km}$ NE Al Mukala, $20 \mathrm{~km}$ NW Ar Rayyan, Al Ain, 100 m, 1.v.1998, leg. M. Fibiger et al., ZMUC; 11 o $^{\top}, 14^{\circ} 46^{\prime} / 49^{\circ} 18^{\prime} 150$ m, Al Ain, Al Mukalla, 14.xi.1996, leg. H. Hacker, ZMHB; SDEI; $10^{\star}, 13^{\circ} 09^{\prime} / 45^{\circ} 19^{\prime}, 80 \mathrm{~m}$, 50 km NE Aden, 16.xi.1996, leg. H. Hacker, ZMHB:

First country records. Hitherto known from Saudi Arabia, the UAE and from Iran.

\section{Pachyarthra africana sp. n.}

Holotype: $o^{\text {đa }, ~ " S u d a n, ~ S i n k a t, ~ 29 . x .1962, ~[l e g .] ~ S . ~ P a n e l i u s ; " ~}$ "Gen.präp.[genitalia slide] Gaedike Nr. 5984;" "Holotypus $0^{*}$, Pachayrthra africana sp. n., det. R. Gaedike 2013;” FMNH.

Derivatio nominis: Named after the continent where the holotype was collected.

Diagnosis (Fig. 8): Wingspan $12 \mathrm{~mm}$; head brush pale creamy; antennae thick, grey-brown, scapus with same coloration as head brush; labial palpi short, inside pale creamy, outside grey-brown; thorax and tegulae grey-brown; forewings nearly completely covered by grey-brown scales, only a stripe from base below cell to $2 / 3$ yellowish brown, ending in a pale creamy dot at beginning of fringe; some light brown scales on the area before apex; hindwings dark grey.

Male genitalia (Fig. 39): Uncus with two thin lobi (one of them destroyed), gnathos arms narrow, with rounded tip; tegumen band-shaped, vinculum broad, without saccus; valvae broad, first half parallel-sided, then abruptly narrower to pointed apex; inside the valvae an oblique edge from $1 / 2$ of ventral edge to a rounded flap; basal edge more strongly sclerotised, apical half with bristled costal and ventral edge, phallus pistol-shaped, basal half with sclerotised edges, apical half deeply incised.

\section{Female genitalia: Unknown.}

Remarks: The new species is the first member of this genus in the Afrotropical Region. The distribution area of the genus covers the North African countries, the Near and Middle East, eastwards to Nepal. 


\section{Ateliotum parvum Petersen, 1988}

Sudan: Petersen, 1988.

New records: 2 ơ, Ed Damer, Hudeiba, 2., 22.v.1962, leg. R. Remane; ZSM.

Hitherto known only from Sudan.

The location of the holotype: "Hudeiba, light trap" was misinterpreted in the original diagnosis as an Iranian locality.

\section{Ateliotum arabicum Petersen, 1961}

Saudi Arabia: Petersen, 1961a; Petersen \& Gaedike, 1982.

Oman: 1 , Northern Region, $20 \mathrm{~km}$ SE Ibra, 300 m, 11.i.1993, leg. B. Skule, ZMUC; 1 o $^{\star}$, Northern Region, Jabal Shams, 19 km NW Al Hamra, 1100 m, 7.i.1993, leg. B. Skule, ZMUC:

First country records.

Yemen: Petersen, 1961a.

New records: $1 \mathrm{o}^{\top}, 15 \mathrm{~km}$ se Taizz, $1350 \mathrm{~m}, 13^{\circ}, 28^{\prime} / 44^{\circ}, 09^{\prime}$ 19.xi.1996, leg. H. Hacker, ZMHB; 2 ơ $^{\star}$ Ma’rib, Ma’rib, 24.iv.1992, leg. R. Linnavuori, FMNH.

Sudan: 3 ơ, Ed Damer, Hudeiba, 20.xii.1961, 23., 28.ii. 1962, leg. R. Remane; ZSM:

First country records. Hitherto known from Saudi Arabia, Yemen, Iran and Afghanistan.

\section{Syncalipsis sudanica GozMÁNy, 1965}

Sudan: GoZMÁNY \& VÁRI, 1973.

Hitherto known only from the holotype from Sudan.

\section{Machaeropteris baloghi GozMÁnY, 1965}

Sudan: GoZMÁNY \& VÁRI, 1973.

Hitherto known from Congo and from Sudan.

\section{Phthoropoea carpella WaLSINGHAM, 1896}

Yemen: Walsingham \& Hampson, 1896.

New records: 5 đ , Hudaydah, Bajil, 19.iii.1992, leg. R. Linnavuori, FNMH; SDEI; 1 ㅇ, Shabwah, Mayfa'ah, 7.iv.1992, leg. R. Linnavuori, FNMH; 1 ㅇ, Prov. Shabwah, 5 km SE Mayfa'ah, 440 m, 30.iv.1998, leg. M. Fibiger et al.,

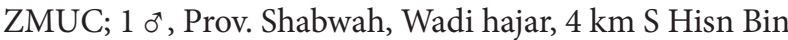
Tallb, Delta, 3 m, 3.v.1999, leg. M. Fibiger et al., ZMUC; 1 ơ, Prov. Ibb, 5 km NE Qa'jdah, 1850 m, 28.iv.1998, leg. M. Fibiger et al., ZMUC:

Hitherto known only from Aden (holotype).

Bionomics: The holotype was reared from fruits of Solanum spec. (GOZMánY \& VÁRI, 1973).

\section{Perissomasticinae GoZMÁNY, 1965}

Edosa lardatella (LEDERER, 1858)

Saudi Arabia: Petersen, 1961b (under the name Episcardia lardatella).

Yemen: $20^{*}$, Wadi Daw'an, Al Huraydah, $900 \mathrm{~m}$, $15^{\circ} 24^{\prime} / 48^{\circ} 21^{\prime}, 13 . x i .1996$, leg. H. Hacker, ZMHB; 1 o $^{*}$, Prov. Shabwah, Abadella Garib Plateau, $63 \mathrm{~km}$ NW Mukalla, 1335 m, 2.v.1999, leg. M. Fibiger et al., ZMUC; $10^{\star}$, Prov. Shabwah, $14^{\circ} 03^{\prime} 49^{\prime \prime N}, 48^{\circ} 41^{\prime} 56^{\prime \prime E}$, Wadi Hajar, $4 \mathrm{~km}$ s Hisn Bin Talib, delta, 3.v.1998, leg. H. Hacker et al., coll. Derra:

First country records. Hitherto known from the entire North African region, as well as the Arabian Peninsula, Turkey and Iran.

\section{Edosa amseli (Petersen \& Gaedike, 1982)}

Saudi Arabia: Petersen \& Gaedike, 1982 (under the name Episcardia amseli).

Hitherto known only from Saudi Arabia.

\section{Edosa islamella (Petersen \& GAEDIKE, 1982)}

Saudi Arabia: Petersen \& Gaedike, 1982 (under the name Neoepiscardia islamella).

Yemen: $3 \sigma^{-1}$, Prov. Sana'a, $60 \mathrm{~km}$ SW Makaban, Naqil Manakhah, $1730 \mathrm{~m}, 21 . i v .1998$, leg. M. Fibiger et al., ZMUC; SDEI; 1 ㅇ, Prov. Sana’a, 15ํ12'64"N, 4357'38"E, 6 km NW Suq Baw’an, 3000 m, 20.iv.1998, leg. M. Fibiger, ZMUC; $80^{\pi}, 6$ 우, Ibb, $1,5 \mathrm{~km}$ W Jiblah, $2100 \mathrm{~m}$, $13^{\circ} 57^{\prime} / 43^{\circ} 57^{\prime}, 7 . x i .1996$, leg. H. Hacker, ZMHB; SDEI; 1 우, Prov. Ibb, $14^{\circ} 02^{\prime} 78^{\prime \prime} \mathrm{N}, 44^{\circ} 11^{\prime} 87^{\prime \prime} \mathrm{E}$, Wadi Malhama, Village Walhama, 20 km NON Ibb, 1650 m, 6.v.1998, leg. Hacker et al., Coll. Derra; 1 ㅇ, Prov. Al Hudaydah, Jabel, Burra, 25 km SE Bajil, 600 m, 23.-24.iv.1998, leg. M. Fibiger et al., ZMUC; $20^{\circ}, 1$ ㅇ, $15 \mathrm{~km}$ SE Taizz, $1350 \mathrm{~m}, 13^{\circ}, 28^{\prime} / 44^{\circ}, 09^{\prime}$, 19.xi.1996, leg. H. Hacker, ZMHB:

First country records. Hitherto known only from Saudi Arabia (type locality).

\section{Edosa pareffulgens sp. n.}

Holotype: $0^{\star}$, "Yemen, Prov. Sana’a, 60 km SW Makaban, Naqil Manakhah, 1730 m, 21.iv.1998, leg. M. Fibiger et al.;" "Coll. ZMUC, Copenhagen Denmark;" "Gen.präp. [genitalia slide] Gaedike Nr. 6053;" "Holotypus ō, Edosa pareffulgens sp. n., det. R. Gaedike 2013;" ZMUC.

Derivatio nominis: Named after the similarity to E. effulgens Gozmany, 1965.

Diagnosis (Fig. 9): Wingspan ca. $12 \mathrm{~mm}$ (specimen incompletely spread); head brush light yellow, between antennae darker yellow; antennae pale creamy; labial palpi short, straight, with grey-brown scales; thorax and tegulae dark brown, edge to abdomen clay-coloured, with 
same colouration as the forewings; forewings without any pattern, the first third of costal edge dark brown; hindwings dark grey.

Male genitalia (Fig. 40): Uncus divided into two lobes, the outer edge strongly sclerotised, apically narrower with bent tip; vinculum broad, ventrally narrower; valvae with bifurcate apodemes, first half nearly square, costal edge curved, ending in a thin finger-like process, ventral edge more or less straight, apically clearly longer than the finger-like costal process; phallus basally broad, fused with vinculum, apically narrower, with strongly sclerotised edges, apex truncated.

Female genitalia: Unknown.

Remarks: Superficially not distinguishable from the other members of the genus. In male genitalia similar to E. effulgens, but the valvae with curved costal edge and without hook are clearly characteristic for this species.

\section{Edosa albicapitella sp. n.}

Holotype: $0^{\star}$, "Yemen, prov. Shabwah Abdalla Garib Plateau, 63 km NW Mukalla, 2.v.1999, 1335 m, leg. M. Fibiger et al.;" "Coll. ZMUC, Copenhagen Denmark;" "Gen.präp.[genitalia slide] Gaedike Nr. 6065;" "Holotypus $\sigma^{\star}$, Edosa albicapitella sp. n., det. R. Gaedike 2013;” ZMUC.

Derivatio nominis: Name refers to the coloration of the head brush.

Diagnosis (Fig. 10): Wingspan $9 \mathrm{~mm}$; head brush white; antennae pale creamy; labial palpi short, with grey-brown scales; thorax and tegulae grey-brown, edge of abdomen paler; forewings yellowish-creamy, without any pattern; hindwings shiny light grey.

Male genitalia (Fig. 41): The two lobes of uncus contorted in the middle, with pointed tip; vinculum broad bandshaped; valvae with long truncated apodemes, square, ventral edge in the second third narrow, bristled, fingerlike, above, at apical edge, with thin pointed process; phallus fused with vinculum, more or less parallel-sided, apically truncated.

\section{Female genitalia: Unknown.}

Remarks: Superficially similar to preceding species, but with white head brush; in male genitalia the species is distinguishable by the contorted uncus lobes and the shape of valvae.

\section{Edosa furcata sp. n.}

Holotype: ${ }^{\star}$, "Yemen, Prov. Al Hudaydah, Jabel Burra, 25 km SE Bajil, 600 m, 23.-24.iv.1998, leg. M. Fibiger et al.;" "Coll. ZMUC, Copenhagen Denmark;" "Gen. präp.[genitalia slide] Gaedike Nr. 6049;" "Holotypus o’, Edosa furcata sp. n., det. R. Gaedike 2013;" ZMUC; Paratype: $1 \sigma^{\star}$, "Sudan, Ed Damar, Hudeiba, 20.vi.1962, leg. R. Remane, Staatss[amm]1[un]g München;" “Gen.präp. [genitalia slide] Gaedike Nr 5992;" ZSM.

\section{Derivatio nominis: Named after the shape of uncus.}

Diagnosis (Fig. 11): Wingspan $10 \mathrm{~mm}$; head brush lemon-yellow; antennae nearly white; labial palpi short, brown; thorax and tegulae and forewings brown; forewings without any pattern, apical half of fringe dark brown; hindwings dark grey.

Male genitalia (Fig. 42): Uncus bifurcate, with pointed tips; tegumen and vinculum broad band-shaped, distal edge of vinculum more strongly sclerotised; valvae lanceolate, basally with narrow apodeme and short square process, broadest at first third, costal edge straight, ventral edge becoming narrower to rounded apex; inside, in the first third, from base between apodeme and process, a thin rod-shaped sclerotisation; phallus connected with tegumen, hook-shaped, basally and laterally with strong sclerotised edges.

\section{Female genitalia: Unknown.}

Remarks: In male genitalia there are similarities to the species of the species group "paraxena" of Gozmány \& VÁRI (1973), but the shape of valvae and the straight rod-shaped sclerotisation inside is different. The lemonyellow head brush is an additional characteristic.

\section{Edosa cristata (GozMÁNY, 1967)}

Sudan: Gozmány \& VÁRI, 1973 (Under the name Sphallesthasis cristata).

Hitherto known only from Sudan, Gambia and Nigeria.

The following genus was previously known only from Afrotropical region, represented by twelve species (GOZMÁny \& VÁRI, 1973). The examination of the material from Yemen and from Oman shows that three species, two of them new, are members of the Palaearctic fauna.

\section{Hyperbola phocina (MeYRICK, 1908)}

Yemen: $1 \sigma^{\star}$, Makaban Naquil Man., $900 \mathrm{~m}, 15^{\circ} 04^{\prime} \mathrm{N}$, 4339'E, 2.xi.1998, leg. H. Hacker, ZMHB; $10^{\top}$, Ibb, 1,5 km W Jiblah, $2100 \mathrm{~m}, 13^{\circ} 57^{\prime} \mathrm{N}, 43^{\circ} 57^{\prime} \mathrm{E}, 7 . x i .1998$, leg. H. Hacker, ZMHB; $30^{*}, 1$ ㅇ, Prov. Al Hudaydah, Jabel, Burra, 25 km SE Bajil, 600 m, 23.-24.iv.1998, leg. M. Fibiger et al., ZMUC; SDEI; 1 ㅇ, Prov. Ibb, 5 km NE Al Qa'jdah, 1850 m, 28.iv.1998, leg. M. Fibiger et al., ZMUC; $10^{\star}$, Sana’a, March 1992, leg. R. Linnavuori, FMNH; $10^{\star}$, Prov. Ibb, Wadi Walhama, Village Walhama, $1650 \mathrm{~m}, 14^{\circ} 02^{\prime} 78^{\prime \prime} \mathrm{N}, 44^{\circ} 11^{\prime} 57^{\prime} \mathrm{E}, 6 . v .1998$, leg. H. Hacker 
et al., coll. Derra; $20^{\star}, 15 \mathrm{~km}$ SE Taizz, $1350 \mathrm{~m}, 13^{\circ} 28^{\prime} \mathrm{N}$, 440'ㄹ, 19.xi.1996, leg. H. Hacker, ZMHB; SDEI:

First records outside the African continent. Hitherto known from Afrotropical region (GOZMÁNY \& VÁRI, 1973).

\section{Hyperbola lehmanni sp. $\mathrm{n}$.}

Holotype: $0^{\star}$, "Oman, mer. Prov. Dhofar, $19 \mathrm{~km} \mathrm{~W}$ Al-Mughhsayl, 790 m, N 1650'27,4", E 5340'78,5", L. Fallen [light traps], 23.-24.xii.2009, leg. L. Lehmann, Bittner \& Stadie;" "Holotypus o*, Hyperbola lehmanni sp. n., det. R. Gaedike 2013;" SDEI; Paratypes: 3 o", 1 ㅇ, same location; "Gen.präp.[genitalia slide] Gaedike Nr. 7934; 7959;" coll. Stübner; SDEI; 2 o $^{\star}$, “Oman, mer. Prov. Dhofar, ca. 8 km W Al-Mughhsayl, 80-100 m, N 1651'99,3", E 53²4'91,1", L. Fallen [light traps], 23.-24. xii.2009, leg. L. Lehmann, Bittner \& Stadie;“"coll. Stübner; $10^{\star}, 1$ ㅇ , "Oman, mer. Prov. Dhofar, ca. 10 km N Salalah, $350 \mathrm{~m}$, Wadi hahiz, Trockenwald, N 17²10'52,5",E

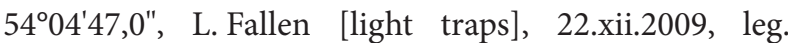
L. Lehmann, Bittner \& Stadie;" "Gen.präp.[genitalia slide] Gaedike Nr. 7931;” coll. Stübner; SDEI.

Derivatio nominis: Named in memory of the collector, Lutz Lehmann, who died during a collecting trip in Oman.

Diagnosis (Fig. 12): Wingspan 10-13 mm; head brush whitish, above palpi pale yellowish; antennae light greybrown, scapus darker, with pecten; labial palpi short, light grey-brown, segments apically darker; thorax and tegulae shiny light yellowish brown; forewings with same coloration, without any pattern; hindwings shiny whitish.

Male genitalia (Figs 43-44): Uncus with two lobes, each with two strongly sclerotised thorns, the basal thorns two times longer than the apical thorns; tegumen and vinculum forming a capsule, phallus fused with vinculum by a finger-like process, nearly pistol-shaped, obliquely truncated; valvae oval, basal edge, apodeme and first third of costal edge more strongly sclerotised, costal edge ending in a short pointed tip, ventral edge evenly narrower to apex.

Female genitalia (Fig. 42): Anterior apophysae forked, ventral branch enlarged, with numerous fine spines, ostium oval, with strongly sclerotised edges.

Remarks: The shape of uncus lobes with the thorns and the shape of valvae with pointed costal tip are characteristic for this new species.

\section{Hyperbola sexspinosa sp. n.}

Holotype: ơ, "Yemen, 7.xi.1996, 13.57/43.57, 2100 m, Ibb, 1,5 km w Jiblah, leg. H. Hacker;" "Gen.präp.[genitalia slide] Gaedike Nr. 8083;" "Holotypus ơ, Hyperbola sexspinosa sp. n., det. R. Gaedike 2013;" ZMHB; Paratype: 1 o", "Yemen, 6.xi.1996, 13.45/44.10, Al Qu-idah, Mahal al Houm, leg. H. Hacker, 1800 m;" "Gen.präp. [genitalia slide] Gaedike Nr. 5994;" "Paratypus o, Hyperbola sexspinosa sp. n., det. R. Gaedike 2013;” SDEI.

Derivatio nominis: Name refers to the six thorns on uncus lobes.

Diagnosis (Fig. 13): Wingspan ca. $15 \mathrm{~mm}$ (specimen not spread); head brush light yellow, scape with pecten dark grey; labial palpi short, straight, outside dark grey, inside paler; thorax and tegulae grey-brown, apically paler, forewings yellowish brown, without any pattern, base and first third of costa darker; hindwings shiny light grey.

Male genitalia (Fig. 46): Uncus with two lobes, each with three strongly sclerotised thorns; tegumen with more strongly sclerotised basal and apical edges; phallus fused with vinculum by finger-like process, nearly pistolshaped, ventral edge prolonged to thin pointed tip, costal edge strongly sclerotised; valvae more or less triangular, basally broadest, narrower to rounded apex, apodemes long, costal edge slightly concave, more strongly sclerotised.

Female genitalia: Unknown.

Remarks: Superficially not clearly distinguishable from the preceding species, but the male genitalia with six thorns on the uncus lobes (four in H. lehmanni) and the rounded apex of valvae without pointed tip are characteristic for this species.

\section{Malacyntis stibarodes MeYrick, 1908}

Sudan: 4 o $^{\star}$, Sudan Equatoria. Mwolo-Mundri, 24.ii.1963, leg. Linnavuori; FMNH:

First country records. Hitherto known from Sierra Leone, Nigeria and Ghana (Gozmány \& Vári, 1973).

\section{Perrissomastix (Aphrodoxa) flava (Petersen, 1960)}

Saudi Arabia: Petersen, 1961b (under the name Catabola flava).

Bahrain Islands: Petersen, 1964 (under the name Catabola flava).

Yemen: $10^{\top}$, Hawra Wadi Hadramaut, $15^{\circ}, 44^{\prime} \mathrm{N}, 48^{\circ}, 18^{\prime} \mathrm{E}$, 12.xi.1996, leg. H. Hacker, ZMHB:

First country record. Hitherto known from the Arabian Peninsula and from Iran.

\section{Perissomastix (Perissomastix) wadimaidaq GAEDIKE, 2009}

UAE: GAEDIKE, 2009.

Oman: GAEDIKe, 2009. 
New records: $20^{*}, 1$ ㅇ Prov. Batinah, Al-Jabal al Ahdar Mts, Wadi Bani, $430 \mathrm{~m}, 23^{\circ} 20^{\prime} 0,13^{\prime \prime} \mathrm{N}, 57^{\circ} 29^{\circ} 23,5^{\prime \prime} \mathrm{E}$, 19.-20.xii.2009, leg. L. Lehmann et al., coll. Stübner; SDEI; $320^{\star}$, Prov. Batinah, Al-Jabal al Ahdar Mts, SE Rustaq, W Awabi, $430 \mathrm{~m}, 23^{\circ} 20^{\prime} 0,13^{\prime \prime} \mathrm{N}, 57^{\circ} 29^{\circ} 23,5^{\prime \prime} \mathrm{E}$, 19.-20.xii.2009, leg. L. Lehmann et al., coll. Stübner; SDEI; $20^{\star}$, Prov. Al-Sharqlyah, $36 \mathrm{~km}$ SE Sur, $26 \mathrm{~m}, 9 \mathrm{~km}$ S Ras al-Hadd, ca. $2 \mathrm{~km}$ inland from turtle Beach, 22²6'19,4"N, 4948'99,2"E, 28.xii.2009, leg. L. Lehmann et al., coll. Stübner; $1 \sigma^{*}$, Prov. Dhofar, $8 \mathrm{~km}$ W Al-Mughsayl, 80-100 m, 16²51'99,3"N, 5342'91,1"E, 23.-24.xii.2009, leg. L. Lehmann et al., SDEI; $10^{\star}$, Prov. Dhofar, $18 \mathrm{~km} \mathrm{~W} \mathrm{Al-Mughsayl,} 717 \mathrm{~m}, 16^{\circ} 50^{\prime} 70,6^{\prime \prime} \mathrm{N}$, 5341'15,6"E, 25.xii.2009, leg. L. Lehmann et al., coll. Stübner; $10^{\top}$, Prov. Dhofar, $19 \mathrm{~km} \mathrm{~W} \mathrm{Al-Mughsayl,}$ $790 \mathrm{~m}, 16^{\circ} 50^{\prime} 27,4^{\prime \prime} \mathrm{N}, 53^{\circ} 40^{\prime} 78,5^{\prime \prime} \mathrm{E}, 23 .-24 . x i i .2009$, leg. L. Lehmann et al., coll. Stübner.

Yemen: Gaedike, 2009.

Hitherto known only from the Arabian Peninsula.

As the series contains a female, it is possible to describe the female genitalia for the first time (Figs 47-48): Anterior apophysae forked, ventral branches bristled, band-shaped connection around ostium, dorsal branches ending in more or less triangular plates, with strongly sclerotised bristles; papillae anales are a plate, basally narrow, ending broader with a finger-like and a rounded process. Possesses the bristled pads at base of papillae which are characteristic for the genus.

\section{Perissomastix (Perissomastix) versicolor GAEDIKE, 2009}

UAE: GAEDIKE, 2009.

Oman: GAEDIKE, 2009.

New record: $1 \sigma^{\star}$, Oman sept. or., Prov. Al-Aharqlyeh, $36 \mathrm{~km}$ SE Sur, 22²6'19,4"N, 5948'99,2"E, 28.xii.2009, leg. L. Lehmann et al., coll. Stübner.

Yemen: Gaedike, 2009.

New record: $10^{\top}, 14^{\circ} 46^{\prime} \mathrm{N}, 49^{\circ} 18^{\prime} \mathrm{E}, 25 \mathrm{~km}$ nne Al Mukalla, Al Ain 20 km NNW Ar Rayan, 14.xi.1996, 150 m, leg. H. Hacker et al., coll. Derra.

Hitherto known only from the Arabian Peninsula.

\section{Perissomastix (Perissomastix) biskraella (REBEL, 1901)}

Yemen: 7 o`, $15 \mathrm{~km}$ se Taizz, $1350 \mathrm{~m}$, 19.xi.1996, 13² $28^{\prime} \mathrm{N}$, $44^{\circ} 09^{\prime} \mathrm{E}$, leg. H. Hacker, ZMHB; SDEI:

First country records. Hitherto known from Mauretania, Morocco, Algeria, Tunisia, Lybia, Egypt, Jordan, Israel and Turkey.

\section{Perrissomastix (Perissomastix) nigriceps WaRREN \& ROTHSCHILD, 1905}

Saudi Arabia: Petersen, 1961a (under the name Catabola intermediella); Petersen \& GAEDIKe, 1982.

Yemen: 1 o $^{\star}$, Prov. Al Hudaydah, Jabel, Burra, 25 km SE Bajil, 600 m, 23.-24.iv.1998, leg. M. Fibiger et al., ZMUC; $30^{\prime}$, Prov. Sana’a, $15^{\circ} 18^{\prime} 12^{\prime \prime N}, 44^{\circ} 16^{\prime} 48^{\prime \prime E}$, mountains SW Sana’a, Jabal 'Ayban, Bait Na’arna, 2700 m, 18.iv.1998, leg. M. Fibiger et al., ZMUC; SDEI, coll. Derra; $10^{\star}$, Prov, Ibb, 5 km NON Al Qa'idah, 1840-1900 m, 28.iv.1998, leg. H. Hacker et al., coll. Derra:

First country records.

Sudan: GoZMÁnY \& VÁRI, 1973.

Hitherto known from Saudi Arabia, Jordan and Sudan.

\section{Perissomastix (Perissomastix) taeniaecornis (WALSINGHAM, 1896)}

Saudi Arabia: Petersen \& Gaedike, 1982.

Oman: $50^{\star}$, Prov. Batinah, Al-Jabal al Ahdar Mts, Wadi Bani, $430 \mathrm{~m}, \quad 23^{\circ} 20^{\prime} 0,13^{\prime \prime} \mathrm{N}, \quad 57^{\circ} 29^{\circ} 23,5^{\prime \prime} \mathrm{r}, 19 .-20$.xii. 2009, leg. L. Lehmann et al., coll. Stübner; SDEI; $20^{\star}$, Prov. Dhofar, ca. $10 \mathrm{~km} \mathrm{~N}$ Salalah, $350 \mathrm{~m}$, Wadi hahiz, Trockenwald, $17^{\circ} 10^{\prime} 52,5^{\prime \prime} \mathrm{N}, 54^{\circ} 04^{\prime} 47^{\prime \prime E}$, 22.xii.2009, leg. L. Lehmann et al., coll. Stübner; SDEI; $2 \sigma^{\star}$, Prov. Al-Dakhilliyah, Passhöhe $1810 \mathrm{~m}, \mathrm{~N}$ Birkat Al Sharaf, Al Alamayn, $23^{\circ} 10^{\prime} 09,7^{\prime \prime} \mathrm{N}, 57^{\circ} 25^{\prime} 82,4^{\prime \prime E}, 21 . x i i .2009$, leg. L. Lehmann et al., coll. Stübner; SDEI; $70^{\star}$, Prov. Dhofar, $8 \mathrm{~km} \mathrm{~W}$ Al-Mughasayl, $80-100 \mathrm{~m}, \quad 1^{\circ} 51^{\prime} 99,3^{\prime \prime} \mathrm{N}, \quad 53^{\circ} 42^{\prime} 91,1^{\prime \prime} \mathrm{E}$, 23.-24.xii.2009, leg. L. Lehmann et al., coll. Stübner; SDEI: First country records.

Yemen: $20^{\star}$, Bajil Khamis Bani Sa'd, $750 \mathrm{~m}, 15^{\circ}, 11^{\prime} / 43^{\circ}, 32^{\prime}$, 3.xi.1996, leg. H. Hacker, ZMHB; $30^{*}$, Al Quidah, Mahal al Houm, $1800 \mathrm{~m}, 13^{\circ}, 45^{\prime} / 44^{\circ}, 10^{\prime}$, 6.xi.1996, leg. H. Hacker, ZMHB; 4 o $^{\star}, 15 \mathrm{~km}$ se Taizz, $1350 \mathrm{~m}, 13^{\circ}, 28^{\prime} / 44^{\circ}, 09^{\prime}$, 19.xi.1996, leg. H. Hacker, ZMHB; 1 \%, Ta’izz, 22.-23.iii. 1992, leg. R. Linnavuori, FNMH; $10^{*}, 50 \mathrm{~km}$ ne Aden, $80 \mathrm{~m}, 13^{\circ}, 09^{\prime} / 45^{\circ}, 1^{\prime}, 16 . x i .1996$, leg. H. Hacker, ZMHB; $190^{\circ}$, Ibb, 1,5 km w Jiblah, $2100 \mathrm{~m}, 13^{\circ} 57^{\prime} / 43^{\circ}, 57^{\prime}, 7 . x i$. 1996, leg. H. Hacker, ZMHB; SDEI; 1 ㅇ, Prov. Ibb, 5 km NE Al Qa’jdah, 1850 m, 28.iv.1998, leg. M. Fibiger et al., ZMUC; 2 ${ }^{\star}$, Prov. Ibb, Wadi Malhama, Village Walhama, $20 \mathrm{~km}$ non Ibb, 1650 m, 6.v.1998, leg. Hacker et al., coll. Derra; 2 ㅇ, Prov. Sana'a, 60 km SW Makaban, Naqil Manakhah, 1730 m, 21.iv.1998, leg. M. Fibiger et al., ZMUC; coll. Derra; $20^{\star}$, Abyan, Al Mahfid, 16.iv.1992, leg. R. Linnavuori, FNMH; SDEI; 1 ๙ , Hudaydah, Zabid, 20.v.1992, leg. R. Linnavuori, FNMH:

First country records.

Sudan: GOZMÁNY \& VÁRI, 1973.

New records: $1 \sigma^{*}$, Sudan, Ed Damer Hudeiba, 2.iii.1962, leg. R. Remane; ZSM; 2 o , Sudan, Sinkat, 28.x.1962, leg. S. Panelius; FMNH; 1 o $^{*} 1$ ㅇ, Sudan, Wadi Halfa, 24., 26.ix.1962, leg. S. Panelius; FMNH.

Hitherto known only from Saudi Arabia, Egypt, Sudan and Ethiopia.

As the series contains females, it is possible to describe the female genitalia for the first time (Fig. 49): Anterior apophysae forked, ventral branches connected through a broad band-shaped plate, surrounding the ostium area; dorsal branches ending in more or less triangular connected plates; apical parts of the plates covered with minute bristles. 


\section{Perissomastix (Perissomastix) asiriella Petersen \&} GAEDIKE, 1982

Saudi Arabia: Petersen \& Gaedike, 1982.

Yemen: $20^{\circ}$, Al Quidah, Mahal al Houm, $1800 \mathrm{~m}$, $13^{\circ}, 45^{\prime} / 44^{\circ}, 10^{\prime}$, 6.xi.1996, leg. H. Hacker, ZMHB; $40^{\text {*, }}$, Ta’izz, Vill. Lagius, $750 \mathrm{~m}, 13^{\circ}, 33^{\prime} / 43^{\circ}, 49^{\prime}$, 18.xi.1996, leg. H. Hacker, ZMHB; SDEI; $10^{\prime \prime}$, Sana’a, Sana’a, May 1992, leg. R. Linnavuori, FNMH; $3 \sigma^{\star}$, Prov. Sana’a, 60 km SW Makaban, Naqil Manakhah, 21.iv.1998, 1730 m, leg. M. Fibiger et al., ZMUC; SDEI; $10^{\text {t}}$, Prov. Ibb, 5 km NON AlQa'idah, 1840-1900 m, 28.iv.1998, leg. Hacker et al., coll. Derra:

First country records. Hitherto known only from Saudi Arabia (type locality).

\section{Perissomastix (Perissomastix) perdita Gozmany, 1965}

Saudi Arabia: Petersen \& Gaedike, 1982 (under the synonymous name Perissomastix minuscula).

Sudan: GOZMÁNY \& VÁRI, 1973.

Hitherto known only from Saudi Arabia and Sudan.

\section{Perissomastix (Perissomastix) tihamaella Petersen \&} GAEDIKE, 1982

Saudi Arabia: Petersen \& Gaedike, 1982.

Sudan: $1 \sigma^{\top}$, Sudan, Sharta, W. Halfa distr., 31.iii.1964, leg. J. Kaisila; FMNH:

First country record. Hitherto known only from Saudi Arabia.

\section{Perissomastix (Perissomastix) jemenitica sp. n.}

Holotype: ơ", "Yemen, Hudaydah, Bajil, 19.iii.1992, [leg.] R. Linnavuori,;"'Gen.präp. [genitalia slide] Gaedike Nr. 5972;" "Holotypus o", Perissomastix jemenitica sp. n., det. R. Gaedike 2013;" FNMH.

Derivatio nominis: Named after the country in which the holotype was collected.

Diagnosis (Fig. 14): Wingspan ca. $15 \mathrm{~mm}$ (specimen not spread); head brush dark brown; antennae and labial palpi light clay brown; thorax, tegulae and forewings light clay brown too, forewings without any pattern; hindwings paler than forewings.

Male genitalia (Figs 50-51): Uncus with two long lobi, basally narrowest, broader to truncated, medially lightly impressed apex, costal edge pointed, directed upwards, ventral edge rounded; first half of costal edge concave, second half straight, ventral edge before rounded apex curved; Valvae lanceolate, costal edge straight, ventral edge curved, apex pointed; phallus U-shaped, dorsal branch with clearly defined pointed tip, hook-shaped directed upwards, ventral branch with rounded apex.
Female genitalia: Unknown.

Remarks: Superficially not distinguishable from the other members of the subgenus, differences are only seen in the genitalia structures. Shape of the lobi of uncus (truncated, medially impressed apex) and the hook-shaped pointed tip of phallus differ from the other members of the subgenus (see Petersen, 1988; GAEDIKE, 2009).

\section{Praelongicera palaestinella AMSEL, 1956}

Saudi Arabia: Petersen \& Gaedike, 1982 (under the name Perissomastix palaestinella).

Oman: $2 \sigma^{\star}$, Northern Region, Jabal Shams, $19 \mathrm{~km}$ NW Al Hamra, 1100 m, 7.i.1993, leg. B. Skule, ZMUC; $50^{\circ}$, Northern Region, Wadi Al Khawd, 100 m, 20 km S Seeb, 12.i.1993, leg. B. Skule, ZMUC; SDEI; $10^{\star}$, Northern Region, Wadi Tanuf, $650 \mathrm{~m}, 8 . i .1993$, leg. B. Skule, ZMUC; $10^{\star}$, Northern Region, Al Hail, $7 \mathrm{~km}$ E Seeb, 30.xii.1992, leg. B. Skule, ZMUC;

$30^{\circ}$, Northern Region, Wadi Abyad, $230 \mathrm{~m}, 23^{\circ}, 25^{\prime} \mathrm{N}$, $57^{\circ}, 04^{\prime}$ E, 21.xii.1994, leg. B. Skule \& M. D. Gallagher, ZMUC; SDEI:

First country records.

Yemen: Petersen, 1961a (under the name Catabola palaestinella).

New records: $1 \sigma^{\top}$, Makaban Naqil Man, $1900 \mathrm{~m}$, $15^{\circ}, 04^{\prime} / 43^{\circ}, 39^{\prime}$, 2.xi.1996, leg. H. Hacker, ZMHB; $10^{\text {* }}$, Al Ain Al Mukalla, $150 \mathrm{~m}, 46^{\circ}, 49^{\prime} / 49^{\circ}, 13^{\prime}$, 14.xi.1996, leg. H. Hacker, ZMHB; $20^{\star}$, Bajil Khamis Bani Sa’ad, 750 m, $15^{\circ}, 11^{\prime} / 43^{\circ}, 32^{\prime}, 3 . x i .1996$, leg. H. Hacker, ZMHB; SDEI; $40^{\star}$, Prov. Táizz, Wadi Warazan, $5 \mathrm{~km}$ NW Ar Rahidah, 1080 m, 27.iv.1998; 1100 m, 26.iv.1998, leg. M. Fibiger et al., ZMUC; SDEI; 1 ơ $^{7}$, Shabwah, Mayfảah, 7.iv.1992, leg. R. Linnavuori, FNMH.

Hitherto known from Saudi Arabia, Oman, Turkey and Iran.

\section{Ectabola pygmina (GozMÁNY, 1965)}

Saudi Arabia: $10^{7}$, SW-Arabien, Asir-Gebirge, $2000 \mathrm{~m}$, Wadi Marah, $81 \mathrm{~km}$ s. Biljurshi, 29.iv.-2.v.1979, leg. H. G. Amsel, SMNK.

Yemen: $2 \sigma^{7}$, Prov. Al Hudaybah, Jebel, Burra, $25 \mathrm{~km} \mathrm{SE}$ Bajil, 23.-24.iv.1998, leg. M. Fibiger et al., ZMUC; SDEI; $60^{\star}$, Prov. Sana’a, Jabal'Ayban, Bait Na’arna, 2700-2750 m, $15^{\circ} 18^{\prime} 12^{\prime \prime N}, 14^{\circ} 16^{\prime} 48^{\prime \prime E}$, 18.iv.1998, leg. M. Fibiger et al., ZMUC; coll. Derra; SDEI; $20^{\circ}$, Ibb, 1,5 km W Jiblah, $2100 \mathrm{~m}, 1^{\circ} 57^{\prime} \mathrm{N}, \quad 43^{\circ} 57^{\prime} \mathrm{E} ， 7 . x i .1996$, leg. H. Hacker, ZMHB; SDEI; $10^{\star}$, Abyan, Al Mahfid, 16.iv.1992, leg. R. Linnavuori, FNMH:

First records for the genus in the Palaearctic. Hitherto known only from Ethiopia (type locality). 


\section{Tineinae LATREILLE, 1810}

\section{Reisserita arabica (PETERSEN, 1961)}

Saudi Arabia: Petersen, 1961b (under the name Fermocelina arabica).

Hitherto known from Saudi Arabia.

\section{Anomalotinea paepalella (WALSINGHAM, 1907)}

Saudi Arabia: Petersen, 1961b (under the name Fermocelina paepalella).

Hitherto known from Algeria, Tunisia and Saudi Arabia.

\section{Trichophaga cuspidata GozMAnY, 1967}

Saudi Arabia: Petersen \& Gaedike, 1982.

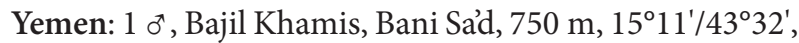
3.xi.1996, leg. H. Hacker, ZMHB:

First country record. Hitherto known from the Afrotropical region (GoZMÁNY \& VÁRI, 1973) and from Saudi Arabia.

\section{Trichophaga bipartitella (RAGONOT, 1892)}

Saudi Arabia: Petersen \& Gaedike, 1982 (under the name Trichophaga abruptella).

New records: $2 \sigma^{\star}$, Djeddah, 16.iii.1979, leg. U. Seneca; ZMUC.

Bahrain Islands: Petersen, 1964 (under the name Trichophaga abruptella).

UAE: GAEDIKE, 2009.

Oman: $1 \sigma^{\star}$, Northern Region, $20 \mathrm{~km}$ SE Ibra, $300 \mathrm{~m}$, 11.i.1993, leg. B. Skule, ZMUC:

First country record.

Yemen: $1 \sigma^{\top}$, Bir Ali, coastal dunes, $10 \mathrm{~m}, 14^{\circ} 01^{\prime} / 48^{\circ} 18^{\prime}$, 15.xi.1996, leg. H. Hacker, ZMHB;

1 ơ, 2 우, Prov. Shabwah, Mayfa’ah, 7.iv.1992, leg. R. Linnavuori, FMNH; $10^{\star}$, Prov. Shabwah, $5 \mathrm{~km} \mathrm{SE} \mathrm{Mayfa'ah,}$ 440 m, 30.iv.1998, leg. M. Fibiger et al., ZMUC:

First country records.

Sudan: Gozmány \& VÁRI, 1973 (under the name Trichophaga abruptella).

Hitherto known from the entire region around the Mediterranean Sea, from the Near and Middle East to the eastern Palaearctic.

\section{Trichophaga atripunctella sp. n.}

Holotype: $0^{\star}$, "Yemen, Prov. Hadramaul, $25 \mathrm{~km} \mathrm{NE}$ Al Mukala, $20 \mathrm{~km}$ NW Ar Rayyan, Al Ain, $100 \mathrm{~m}$, 1.v.1998, leg. M. Fibiger et al.;" "Coll. ZMUC, Copenhagen, Denmark;" "Holotypus o", Trichophaga atripunctella sp. n. det. R. Gaedike 2013;" ZMUC; Paratypes: 1 o $^{*}$, "Yemen, Prov. Shabwah, 5 km SE Mayfa'ah, 30.vi.1998, 440 m, leg. M. Fibiger et al.;" "Coll. ZMUC, Copenhagen, Denmark;" "Gen.präp. [genitalia slide] Gaedike Nr. 8085;" SDEI; 1 ㅇ, same location, same dates, but
“Gen.präp. [genitalia slide] Gaedike Nr. 7908;" ZMUC; $2 \sigma^{\prime}$, "Republik[sic!] of Yemen, $14^{\circ} 16^{\prime} \mathrm{N}, 49^{\circ} 18^{\prime} \mathrm{E}$, $25 \mathrm{~km}$ nne Al Mukalla, Al Ain, $20 \mathrm{~km}$ nnw Ar Rayyan, 14.xi.1996, 150 m, leg. Bischof/Hacker/Schreier;" "Gen. präp. [genitalia slide] Gaedike Nr. 7909;" one specimen with genitalia slide Derra No. 5173, with label: "Genital verloren [genitalia lost]"; coll. Derra; $1 \sigma^{\star}$, same location, but leg. H. Hacker; ZMHB; $10^{*}$, "Yemen, Shabwah, Mayfa’ah, 7.iv.1992, [leg.] R. Linnavuori;” FMNH.

Derivatio nominis: The name refers to the black dot on the forewing.

Diagnosis (Figs 15-16): Wingspan 14-18 mm; head brush white, scape, with pecten, and pedicellus of antennae white, flagellum light brown, with ciliae ( 0.5 of diameter of segments); labial palpi curved upwards, light brown, with some white scales; thorax and tegulae white, apically light brown; forewings bicoloured. Basal third brown, other part white, border between these parts straight from costa to dorsum; at $3 / 4$, in the white area, at the end of cell a black dot; apex and fringe brown, hindwings shiny whitish.

Male genitalia (Figs 52-55): Uncus apically incised, the two short lobi apically rounded; gnathos arms slightly curved, basal edge of tegumen more strongly sclerotised, vinculum with long saccus, proximal edge elongate, nearly triangular; valvae with straight costal edge, ventral edge from apodeme oblique broadened to strongly sclerotised angle at $1 / 2$, from it to rounded apex narrower; inside, parallel to oblique ventral edge some strongly sclerotised wrinkles; subapically, below costal edge a short lobe. Shape of wrinkles and lobe varies (see fig. 55); phallus nearly as long as uncus-tegumen-saccus, basally rounded, slender, apically covered with numerous minute sclerotised thorns.

Female genitalia (Fig. 56): Anterior apophysae unforked, ostium and first part of ductus bursae slightly more strongly sclerotised, area between the apophysae and ostium longitudinally with fine sclerotisations.

Remarks: The black dot makes the new species superficially distinguishable from the other members of the genus.

Trichophaga swinhoei (BUTLER, 1884)

Yemen: GoZMÁNY \& VÁRI, 1973.

\section{Ceratophaga tragoptila (MEYRICK, 1917)}

Sudan: Gozmány \& VÁRI, 1973 (under the name Ceratophaga vastella (ZELLER, 1852)).

Hitherto known from nearly the entire Afrotropical region, in the Palaearctic known from Turkey. 


\section{Tinea murariella StAUDINGER, 1859}

Yemen: Petersen, 1961a.

Hitherto known from nearly the entire West Palaearctic region.

\section{Tinea messalina RoBINSON, 1979}

Yemen: Robinson, 1969.

Hitherto known from Algeria, Iberian Peninsula, Italy, Malta, Greece and Yemen.

\section{Tinea subalbidella StAINTON, 1867}

UAE: GAEDIKE, 2009.

Yemen: $1 \sigma^{\star}$, Prov. Ma’rib, Ma’rib, 24.iv.1992, leg. R. Linnavuori, FMNH; $1 \sigma^{\star}$, Sana's, iii.1992, leg. R. Linnavuori, FMNH:

First country records. Hitherto known only from Egypt, the UAE and Pakistan.

\section{Niditinea tugurialis (MEYRICK, 1932)}

Bahrain Islands: Petersen, 1964.

Hitherto known from the southern part of the Palaearctic (from North Africa through Near and Middle East to Far East).

\section{Proterospastis cornuta sp. n.}

Holotype: $0^{*}$, "Yemen, Ta’izz, Taizz, 22.-23.iii.1992, [leg.] R. Linnavuori;" "Holotypus $\sigma^{\star}$, Proterospastis cornuta sp. n., det. R. Gaedike 2013;" FNMH; Paratypes: $1 \sigma^{*}$, same location, "Gen.präp. [genitalia slide] Gaedike Nr. 5978;” FNMH; 1 ơ $^{\star}$, same location; SDEI.

Derivatio nominis: Name refers to the existence of cornuti.

Diagnosis (Fig. 17): Wingspan ca. $10 \mathrm{~mm}$ (specimens not spread); head brush light brown, antennae pale greybrown; labial palpi short, on inside light creamy, on outside darker; thorax and tegulae basally dark brown, apically paler; forewings light brown, more or less, especially in the apical half, overlaid with dark brown scales, forming a stripe at $1 / 2$ from costa obliquely to end of cell; hyaline dot clearly visible; hindwings pale grey-brown.

Male genitalia (Figs 57-58): Uncus truncated, medially incised, tegumen broad, gnathos arms curved, with pointed tip; vinculum narrow, band-shaped, saccus as long as uncus-tegumen, thin; valvae more or less parallel-sided, basal edge more strongly sclerotised, apex bluntly rounded, subapically with a short, strongly sclerotised fold; phallus nearly twice as long as valva, basally rounded, straight, vesica with numerous minute sclerotisations, with two thin cornuti, about a third of the length of phallus.
Female genitalia: Unknown.

Remarks: As the condition of the studied specimens was not optimal, superficially only the clearly visible hyaline spot is remarkable. Characteristic for the new species is the existence of cornuti.

\section{Proterospastis spinosa sp. $\mathrm{n}$}

Holotype: $\sigma^{\star}$, "Yemen, 600 m, Prov. Al Hudaydah, Jabel, Burra, 25 km SE Bajil, 23.-24.iv.1998, [leg.] M. Fibiger et al.;" "Coll. ZMUC Copenhagen Denmark;" "Gen.präp. [genitalia slide] Gaedike Nr. 6048;" "Holotypus o", Proterospastis spinosa sp. n., det. R. Gaedike 2013;" ZMUC; Paratypes: $1 \sigma^{\star}$, "Yemen, Prov. Ibb, 5 km NE Al Qa'jdah, 28.iv.1998, [leg.] M. Fibiger et al.;" "Coll. ZMUC Copenhagen Denmark;" "Gen.präp. [genitalia slide] Gaedike Nr. 6063;" ZMUC; 1 ơ, "Yemen, Ta'izz, Ta’izz, 22.-23. iii.1992, [leg.] R. Linnavuori;" "Gen.präp. [genitalia slide] Gaedike Nr. 5982;" FMNH.

Derivatio nominis: Name refers to the existence of the thorns in the vesica.

Diagnosis (Fig. 18): Wingspan 10-12 mm; head brush pale yellowish; antennae: scape with same colouration, flagellum darker; labial palpi on outside brown, on inside paler, second segment bristled; thorax and tegulae pale yellowish, only basally darker; forewings with a brown pattern on pale yellowish ground color. Brown are oblique stripes from base to dorsum, at $1 / 2$ from costa to dorsum and at $3 / 4$ from costa to dorsum; hindwings grey. Sometimes the entire coloration is darker, the stripes do not reach dorsum, the entire forewing is covered by some dark brown scales and the hindwings are grey-brown.

Male genitalia (Figs 59-61): Uncus truncated, gnathos arms fused, apically narrow, with rounded tip; tegumen narrow, rod-shaped, strongly sclerotised; vinculum and saccus sub-triangular; valvae as long as vinculum-saccus, basal third more or less parallel-sided, then costal edge narrower to rounded apex, ventral edge straight; phallus basally rounded, pistol-shaped, vesica with numerous pointed thorns.

\section{Female genitalia: Unknown.}

Remarks: Superficially similar to $P$. autochthones and P. merdella, but the pointed thorns in the vesica of phallus are clear different in comparision to these two species.

\section{Wyoma striaticostella (PETERSEN, 1959)}

UAE: GAEDIKE, 2009.

Oman: $1 \sigma^{\star}$, Northern Region, $23^{\circ} 28^{\prime} \mathrm{N}, 58^{\circ} 14^{\prime} \mathrm{E}$, Al Hail, 7 km E Seeb, 16.xii.1994, leg. B. Skule, ZMUC:

First country record. Hitherto known only from the UAE and from Irak (type locality). 


\section{Crypsithyris trimaculata (PETERSEN, 1973)}

The re-examination of the specimen from Hudeiba, which was determined as Proterospastis trimaculata in Petersen \& Gaedike (1984), shows that it belongs to C. luteocapitata sp. n., described below.

\section{Crypsithyris luteocapitata sp. n.}

Holotype: ơ, "Staatss[amm]1[un]g München, Sudan, Ed Damer, Hudeiba, 23.v.1962, leg. R. Remane;" “Gen. präp.[genitalia slide] Gaedike Nr. 7910;" "Holotypus ơ, Crypsithyris luteocapitata sp. n., det. R. Gaedike 2013;" ZSM; Paratypes: 4 ơ, same location, but 28.iv., 1.v.1962; ZSM; 29.iv.1962, "Gen.präp.[genitalia slide] Gaedike Nr. 5986;" SDEI; 1 ơ, "Hudeiba, 1.v.1962, lighttrap," “Gen.präp.[genitalia slide] Gaedike Nr. 2225;” SDEI.

Derivatio nominis: Name refers to the colouration of head brush.

Diagnosis (Figs 19-20): Wingspan 7-9 mm; head brush yellowish-brown; antennae on underside pale creamy, on upperside grey-brown; labial palpi short, pale yellowish-whitish, second segment bristled; thorax and tegulae brown, apically paler; forewings yellowish-brown, partly overlaid with brown scales, forming a brown pattern of some patches. More or less clearly visible are patches on dorsum at $1 / 4$ and $1 / 2$, at base on costa and at $2 / 3$ below costa; the apical third more completely covered by brown scales; hindwings grey. Some specimens with paler forewings, the brown pattern of patches more clearly visible (Fig. 20).

Male genitalia (Figs 62-64): Uncus triangular, with pointed tip; gnathos arms ventrally convex, dorsally nearly straight; vinculum narrow, band-shaped, saccus long; valvae as long as uncus-tegumen, slightly curved, apically rounded; phallus longer than valva, nearly straight, thin, vesica with minute sclerotisations.

\section{Female genitalia: Unknown.}

Remarks: Similar to C. trimaculata, described from Afghanistan, but distinguishable superficially from it by a smaller wingspan and by bristled 2nd segment of labial palpi, in the male genitalia phallus is straight and vinculum without additional sclerotisation.

\section{Crypsithyris unipuncta sp. n.}

Holotype: o", "Staatss[amm]l[un]g München, Sudan, Ed Damer, Hudeiba, 24.v.1962, leg. R. Remane;" "Gen. präp.[genitalia slide] Gaedike Nr. 7911;" "Holotypus o", Crypsithyris unipuncta sp. n., det. R. Gaedike 2013;" ZSM; Paratypes: 6 o $^{*}, 2$ 우, same location, but 5.v.1962 (Gen. präp.[genitalia slide] Gaedike Nr. 5991), 8.v.1962, ZSM;
23., 29., 30.v.1962 (Gen.präp.[genitalia slide] Gaedike Nr. 5987, 5989, 5990); SDEI.

Derivatio nominis: Name refers to single dark dot on forewing.

Diagnosis (Fig. 21): Wingspan 5-6 mm; head brush light brown; antennae shiny grey-brown; labial palpi with same colouration, paler inside, second segment with some bristles; thorax and tegulae grey-brown; forewings shiny pale grey-brown, covered with numerous single darker brown scales, forming a dot below costa at end of cell; hindwings grey.

Male genitalia (Figs 65-66): Uncus truncated, gnathos arms basally broad, slightly curved to pointed tip; vinculum band-shaped, laterally each incised, saccus continuously narrowed to rounded tip; valvae lancetshaped, from base to first half of length parallel-sided, then broader to rounded apex, phallus as long as valva, slightly curved, with minute sclerotisations in the vesica.

Female genitalia (Fig. 64): Anterior apophysae unforked, ventrally connected by a band-shaped sclerotisation, covered with many long bristles; ostium slightly funnelshaped, strongly sclerotised, in the ductus bursae a small strongly sclerotised ring.

Remarks: Superficially distinguishable by the very small wingspan and by having only one dot on forewings. In male genitalia characterised by the truncated uncus and vinculum with lateral incisions.

\section{Ceratobia irakella (PETERSEN, 1959)}

UAE: GAEDIKe, 2009.

Oman: $1 \sigma^{\star}$, Northern Region, Jabal Shams, $19 \mathrm{~km}$ NW Al Hamra, 1100 m, 7.i.1993, leg. B. Skule, ZMUC; 1 o, Northern Region, Wadi Tanuf, 650 m, 8.i.1993, leg. B. Skule, ZMUC;

$10^{\star}$, Prov. Batinah, Al-Jabal al Ahbar Mts, SE Rastaq, $430 \mathrm{~m}$, 19.-20.xii.2009, leg. L. Lehmann et al., coll. Stübner; $1 \mathrm{o}^{*}$, Oman, mer. Prov. Dhofar, ca. $8 \mathrm{~km} \mathrm{~W}$ Al-Mughhsayl, 80-100 m, N 1651'99,3", E 5342'91,1", L. Fallen, 23.-24.xii.2009, leg. L. Lehmann, Bittner \& Stadie;" coll. Stübner:

First country records. Hitherto known only from the UAE, Irak and from Iran.

\section{Ceratobia hemiphracta (MeYRICK, 1926), comb. n.}

Yemen: $30^{\star}$, prov. Sana'a, mountains sw Sana’a, Jabal 'Ayban, Bait Na'arna, $2700 \mathrm{~m}, 1^{\circ} 18^{\prime} 12^{\prime \prime N}, 44^{\circ} 16^{\prime} 48^{\prime \prime E}$, 18.iv.1998, leg. M. Fibiger, ZMUC; SDEI:

First record for the Palaearctic, hitherto known only from type location (Zimbabwe: Bulawayo). 
The examination of the male genitalia shows, that the taxon, described in the genus Crypsithyris, belongs to genus Ceratobia Zagulajev, 1974.

\section{Ceratobia sudanica sp. n.}

Holotype: đ^, “[Sudan], Hudeiba, 2.iii.1962, lighttrap, [leg. R. Remane];" "Gen.präp.[genitalia slide] G. Petersen Nr. 2226;" "Holotypus ơ, Ceratobia sudanica sp. n., det. R. Gaedike 2013;" SDEI; Paratype: $1 \sigma^{\star}$, "Staatss[amm] 1[un]g München, Sudan, Ed Damer, Hudeiba, 14.v.1962, leg. R. Remane;" "Gen.präp.[genitalia slide] Gaedike Nr. 5988;" ZSM.

Derivatio nominis: Named after the country, in which were collected the types.

Diagnosis (Fig. 22): Wingspan 5-7 mm; head brush, antennae and labial palpi shiny light yellowish, thorax and tegulae and forewings with same coloration, forewings without any pattern; hindwings nearly white.

Male genitalia (Figs 68-69): Uncus as long as tegumen, with pointed tip; gnathos arms nearly reaching tip of uncus, narrow, slightly curved; vinculum band-shaped, saccus short; valvae longer than uncus-tegumen, costal edge concave, ventral edge at $1 / 2$ slightly bent, apex rounded; phallus a little shorter than valva, slightly curved, thin.

\section{Female genitalia: Unknown.}

Remarks: The first record of the genus from the Afrotropical region.

\section{Monopis imella (HÜBNER, 1813)}

Yemen: $2 \sigma^{\star}$, Prov. Sana'a, mountains sw Sana'a, Jabal 'Ayban, Bait Na’arna, $2700 \mathrm{~m}, 15^{\circ} 18^{\prime} 12^{\prime \prime N}, 44^{\circ} 16^{\prime} 48^{\prime \prime E}$, 18.iv.1998, leg. M. Fibiger, ZMUC; SDEI; $10^{\top}$, Prov. Sana’a, 6 km NW Suq Baw’an, 3000 m, 15²12'64"N, 4357'38"E, 20.iv.1998, leg. M. Fibiger, ZMUC:

First country records. Known from nearly the entire Palaearctic.

\section{Hieroxestinae MEYRICk, 1893}

\section{Amphixystis islamella (TURATI, 1927)}

Oman: 1 q, Prov. Dhofar, ca. $10 \mathrm{~km} \mathrm{~N}$ Salalah, $350 \mathrm{~m}$, Wadi hahiz, $17^{\circ} 10^{\prime} 52,5^{\prime \prime} \mathrm{N}, 54^{\circ} 04^{\prime} 47^{\prime} \mathrm{E}, 22 . x i i .2009$, leg. L. Lehmann et al.; coll. Stübner:

First country record.

Yemen: $10^{\star}$, Prov. Sana’a, Mountains SW Sana'a, Jabal 'Ayban, Bait Na'arna, $2700 \mathrm{~m}, 1^{\circ} 18^{\prime} 12^{\prime \prime N}, 44^{\circ} 16^{\prime} 48^{\prime \prime} \mathrm{E}$, 18.iv.1998, leg. M. Fibiger et al., ZMUC; $10^{\star}$, Prov. Sana’a, 60 km SW Makaban Naqil Manakhah, 1730 m, 21.iv.1998, leg. M. Fibiger et al., ZMUC; 1 o , Prov. Shabwah, Abdalla garib Plateau, 63 km NW Mukalla, 1335 m, 2.v.1999, leg. M. Fibiger et al., ZMUC; 1 ơ, Prov. Ibb, $5 \mathrm{~km} \mathrm{NE} \mathrm{Al}$ Qa'jdah, 1850 m, 28.iv.1998, leg. M. Fibiger et al., SDEI: First country records. Hitherto known only from Libya (type location).

\section{Amphixystis minuta sp. $\mathrm{n}$.}

Holotype: ${ }^{\top}$, "Yemen, 600 m, Prov. Al Hudaydah, Jabel, Burra $25 \mathrm{~km}$ SE Bajil, 23.-24.iv.1998, [leg.] M. Fibiger et al.;" "Coll. ZMUC, Copenhagen Denmark;" "Gen. präp.[genitalia slide] Gaedike Nr. 6017;" "Holotypus o", Amphixystis minuta sp. n., det. R. Gaedike 2013;” ZMUC.

Derivatio nominis: Name refers to the small wingspan contrary the other known members of the genus.

Diagnosis (Fig. 23): Wingspan $7 \mathrm{~mm}$; head brush whitish (only some scales remained); antennae nearly completely destroyed, visible are a dark brown scape and some dark brown flagellomeres; labial palpi pale whitish on inside, on outside with dark brown scales; thorax white, tegulae brown; forewings with characteristic pattern of the genus: costal part dark brown from base to beginning of fringe at dorsum, ventral part white, the border between these two areas undulate; hindwings grey.

Male genitalia (Figs 70-72): Tegumen broad, lateral edges strongly sclerotised, uncus with small narrow lobi, gnathos arms with strongly sclerotised, basally broad, narrower to pointed tip, directed obliquely upwards; vinculum narrow, band-shaped, saccus thin; valvae with long apodemes, parallel-sided, with rounded bristled apex, below costal edge from base to $1 / 2$ a sclerotised thin fold; phallus half the length of valva, narrow, straight, anellus shell-like.

Female genitalia: Unknown.

Remarks: The short saccus and short phallus in male genitalia are the distinguishing structures for this new species, beside the small wingspan.

\section{Amphixystis taurus sp. $\mathrm{n}$.}

Holotype: ${ }^{\star}$, "Yemen, Prov. Ta'izz, Wadi Warazan, $5 \mathrm{~km}$ NW Ar Rahidah, 27.iv.1998, 1080 m [leg.] M. Fibiger et al.;" "Coll. ZMUC, Copenhagen Denmark;" "Gen. präp.[genitalia slide] Gaedike Nr. 6033;" "Holotypus o", Amphixystis taurus sp. n., det. R. Gaedike 2013;” ZMUC.

Derivatio nominis: Name refers to the shape of the two gnathos arms.

Diagnosis (Fig. 24): Wingspan $9 \mathrm{~mm}$; head brush pale creamy; antennae with dark grey-brown scape and grey-brown flagellum; labial palpi on inside creamy, on outside darker; thorax creamy, tegulae brown; forewings 
dark brown, from base to beginning of fringe and fringe creamy, forming the characteristic pattern of the genus; hindwings shiny light grey.

Male genitalia (Figs 73-75): Tegumen broad, uncus absent; gnathos arms horn-like, strongly sclerotised, inner side more strongly sclerotised than outer side; vinculum broad, medially immersed, saccus thin, short; valvae with long apodemes, slender, apically rounded; phallus only $1 / 2$ of the length of valva, thin, with one thorn-shaped cornutus.

\section{Female genitalia: Unknown.}

Remarks: The absent uncus, the horn-like gnathos arms and the small thorn-like cornutus are characteristics for this species.

\section{Unattributed to subfamily (according to RoBINSON, 2009)}

\section{Psecadioides stuebneri sp. n.}

Holotype: $0^{\star}$, "Oman mer. Prov. Dhofar, $8 \mathrm{~km} \mathrm{~W} \mathrm{Al}$ Mghsayl, 80-100 m, N 1651'99,3", E 5342'91,1", LF [lux] 23.-24.xii.2009, leg. L. Lehmann, Bittner \& Stadie;" "Holotypus $\sigma^{\star}$, Psecadioides stuebneri sp. n., det. R. Gaedike 2014;" SDEI; Paratypes: $11 \sigma^{\star}$, same location, same dates, three specimens with genitalia slide (R. Gaedike Nr. 7923, 7953, 7954); SDEI; coll. Stübner.

Derivatio nominis: Named after Andreas Stübner, who enabled me to study the Oman tineids.
Diagnosis (Figs 5-6): Wingspan 20-27 mm; head white, scales below antennae obliquely erect, the others directed to palpi; antennae white, scape with long white pecten, flagellomeres on upper side white, the opposite side dark grey, bipectinate, pectinations elongate and ciliate (Fig. 6); labial palpi on inside white, on outside grey; thorax and tegulae pale grey, tips of thorax and tegulae white; forewings pale grey with a pattern of white stripes. One straight stripe from base below costa to $1 / 3$, a curved stripe below costa from $2 / 3$ to apex, a stripe on base of fringe and an upwardly curved stripe from base at dorsum to beginning of fringe; hindwings grey. Some specimens with ground colour on forewings a darker grey.

Male genitalia (Figs 33-35): Uncus large, apically rounded, with minute tip; tegumen broad, vinculum with short pointed saccus; gnathos arms long, apically rounded and fused; valva spatulate, ventral edge at first half straight, second half convexe to rounded apex, costal edge concave; apodeme strongly sclerotised, the sclerotisation prolonged rod-shaped inside the valva; phallus shorter than valva, slightly curved, apically incised, one side pointed, other side more or less rounded, with some minute teeth.

\section{Female genitalia: Unknown.}

Remarks: Superficially characterised by the bipectinate antennae.

\section{Philagraulella punica (MEYRICK, 1930)}

Sudan: GoZMÁNY \& VÁRI, 1973.

Hitherto known only from the holotype from Sudan.

Checklist of the Tineidae of the southern Arabian Peninsula (Saudi-Arabia - Arab; Katar - Kat; UAE; Bahrain - Bahr; Oman - Om; Yemen - Yem and of Sudan - Sud).

First country record:

\begin{tabular}{|c|c|c|c|c|c|c|c|}
\hline species & Arab & Kat & UAE & Bahr & Om & Yem & Sud \\
\hline \multicolumn{8}{|l|}{ Hapsiferinae ZaGulaJEV, 1968} \\
\hline Hapsifera luridella ZELLER, 1847 & + & - & - & - & $\bullet$ & + & - \\
\hline Hapsifera punctata Petersen, 1961 & + & - & - & - & - & - & - \\
\hline Hapsifera gozmanyi sp. n. & - & - & - & - & - & $\bullet$ & - \\
\hline Hapsifera rhodoptila MEYRICK, 1920 & - & - & - & - & - & - & + \\
\hline Hapsiferona glareosa (MEYRICK, 1912) & + & - & - & - & - & $\bullet$ & - \\
\hline Hapsiferona arabica GAEDIKE, 2009 & - & - & + & - & - & - & - \\
\hline Hapsiferona jemenitica sp. n. & - & - & - & - & - & $\bullet$ & - \\
\hline Hapsiferona remanei sp. n. & - & - & - & - & - & - & $\bullet$ \\
\hline Scalidomia fetialis (MEYRICK, 1917) & - & - & - & - & - & $\bullet$ & - \\
\hline \multicolumn{8}{|l|}{ Myrmecozelinae ZaGULAJEV, 1958} \\
\hline Heterostasis hackeri sp. n. & - & - & - & - & - & $\bullet$ & - \\
\hline
\end{tabular}




\begin{tabular}{|c|c|c|c|c|c|c|c|}
\hline species & Arab & Kat & UAE & Bahr & $\mathrm{Om}$ & Yem & Sud \\
\hline Pachyarthra ochroplicella (CHRÉTIEN, 1915) & - & - & - & - & - & $\bullet$ & - \\
\hline Pachyarthra iranica GAEDIKE, 2009 & - & - & + & - & $\bullet$ & - & - \\
\hline Pachyarthra grisea PETERSEN \& GAEDIKe, 1982 & + & - & + & - & - & $\bullet$ & - \\
\hline Pachyarthra africana sp. $\mathrm{n}$. & - & - & - & - & - & - & $\bullet$ \\
\hline Ateliotum parvum Petersen, 1988 & - & - & - & - & - & - & + \\
\hline Ateliotum arabicum Petersen, 1961 & + & - & - & - & $\bullet$ & + & $\bullet$ \\
\hline Syncalipsis sudanica GoZMÁnY, 1965 & - & - & - & - & - & - & + \\
\hline Machaeropteris baloghi GozmánY, 1965 & - & - & - & - & - & - & + \\
\hline Phthoropoea carpella Walsingham, 1896 & - & - & - & - & - & + & - \\
\hline \multicolumn{8}{|l|}{ Perissomasticinae GozMánY, 1965} \\
\hline Edosa lardatella (LEDERER, 1858) & + & - & - & - & - & $\bullet$ & - \\
\hline Edosa amseli (PETERSEn \& GAEDike, 1982) & + & - & - & - & - & - & - \\
\hline Edosa islamella (Petersen \& GAEDike, 1982) & + & - & - & - & - & $\bullet$ & - \\
\hline Edosa pareffulgens sp. $\mathrm{n}$. & - & - & - & - & - & $\bullet$ & - \\
\hline Edosa albicapitella sp. $\mathrm{n}$. & - & - & - & - & - & $\bullet$ & - \\
\hline Edosa furcata sp. n. & - & - & - & - & - & $\bullet$ & $\bullet$ \\
\hline Edosa cristata (GozmánY, 1967) & - & - & - & - & - & - & + \\
\hline Hyperbola phocina (MEYRICK, 1908) & - & - & - & - & - & $\bullet$ & - \\
\hline Hyperbola lehmanni sp. n. & - & - & - & - & $\bullet$ & - & - \\
\hline Hyperbola sexspinosa sp. $\mathrm{n}$. & - & - & - & - & - & $\bullet$ & - \\
\hline Malacyntis stibarodes MEYRICK, 1908 & - & - & - & - & - & - & $\bullet$ \\
\hline Perrissomastix flava (Petersen, 1960) & + & - & - & + & - & $\bullet$ & - \\
\hline Perissomastix wadimaidaq GAEDIKe, 2009 & - & - & + & - & + & + & - \\
\hline Perissomastix versicolor GAEDIKE, 2009 & - & - & + & - & + & + & - \\
\hline Perissomastix biskraella (REBEL, 1901) & - & - & - & - & - & $\bullet$ & - \\
\hline Perrissomastix nigriceps WARREN \& ROTHSCHILD, 1905 & + & - & - & - & - & $\bullet$ & + \\
\hline Perissomastix taeniaecornis (WALsIngham, 1896) & + & - & - & - & $\bullet$ & $\bullet$ & + \\
\hline Perissomastix asiriella Petersen \& GAedike, 1982 & + & - & - & - & - & $\bullet$ & - \\
\hline Perissomastix perdita Gozmany, 1965 & + & - & - & - & - & - & + \\
\hline Perissomastix tihamaella Petersen \& GAedike, 1982 & + & - & - & - & - & - & $\bullet$ \\
\hline Perissomastix jemenitica sp. $\mathrm{n}$. & - & - & - & - & - & $\bullet$ & - \\
\hline Praelongicera palaestinella AMsEL, 1956 & + & - & - & - & $\bullet$ & + & - \\
\hline Ectabola pygmina (Gozmány, 1965) & * & - & - & - & - & $\bullet$ & - \\
\hline \multicolumn{8}{|l|}{ Tineinae LATREILLE, 1810} \\
\hline Reisserita arabica (Petersen, 1961) & + & - & - & - & - & - & - \\
\hline Anomalotinea paepalella (WALSINGHAM, 1907) & + & - & - & - & - & - & - \\
\hline Trichophaga cuspidata Gozmany, 1967 & + & - & - & - & - & $\bullet$ & - \\
\hline Trichophaga bipartitella (RAGONOT, 1892) & + & - & + & + & $\bullet$ & $\bullet$ & + \\
\hline Trichophaga atripunctella sp. $\mathrm{n}$. & - & - & - & - & - & $\bullet$ & - \\
\hline Trichophaga swinhoei (ButLER, 1884) & - & - & - & - & - & + & - \\
\hline Ceratophaga tragophila (MEYRICK, 1917) & - & - & - & - & - & - & + \\
\hline Tinea murariella StAUdINGER, 1859 & - & - & - & - & - & + & - \\
\hline Tinea messalina RoBInson, 1979 & - & - & - & - & - & + & - \\
\hline
\end{tabular}




\begin{tabular}{|c|c|c|c|c|c|c|c|}
\hline species & Arab & Kat & UAE & Bahr & $\mathrm{Om}$ & Yem & Sud \\
\hline Tinea subalbidella STAINTON, 1867 & - & - & + & - & - & $\bullet$ & - \\
\hline Niditinea tugurialis (MEYRICK, 1932) & - & - & - & + & - & - & - \\
\hline Proterospastis cornuta sp. $\mathrm{n}$. & - & - & - & - & - & $\bullet$ & - \\
\hline Proterospastis spinosa sp. $\mathrm{n}$. & - & - & - & - & - & $\bullet$ & - \\
\hline Wyoma striaticostella (PETERsen, 1959) & - & - & + & - & $\bullet$ & - & - \\
\hline Crypsithyris luteocapitata sp. $\mathrm{n}$. & - & - & - & - & - & - & $\bullet$ \\
\hline Crypsithyris unipuncta sp. n. & - & - & - & - & - & - & - \\
\hline Ceratobia irakella (PETERsen, 1959) & - & - & + & - & $\bullet$ & - & - \\
\hline Ceratobia hemiphracta (MEYRICK, 1926), comb. n. & - & - & - & - & - & $\bullet$ & - \\
\hline Ceratobia sudanica sp. n. & - & - & - & - & - & - & $\bullet$ \\
\hline Monopis imella (HüBNER, 1813) & - & - & - & - & - & $\bullet$ & - \\
\hline \multicolumn{8}{|l|}{ Hieroxestinae MEYRICK, 1893} \\
\hline Amphixystis islamella (TurATI, 1927) & - & - & - & - & $\bullet$ & $\bullet$ & - \\
\hline Amphixystis minuta sp. $\mathrm{n}$. & - & - & - & - & - & $\bullet$ & - \\
\hline Amphixystis taurus sp. $\mathrm{n}$. & - & - & - & - & - & $\bullet$ & - \\
\hline \multicolumn{8}{|l|}{ Unattributed to subfamily } \\
\hline Psecadioides stuebneri sp. $\mathrm{n}$. & - & - & - & - & $\bullet$ & - & - \\
\hline Philagraulella punica (MEYRICK, 1930) & - & - & - & - & - & - & + \\
\hline
\end{tabular}

\section{References}

Gaedike, R. 2009: Order Lepidoptera, family Tineidae. - Arthropod fauna of the UAE 2: 433-444, 10 pls, 23 figs.

GozmánY, L. \& VÁRI, L. 1973: The Tineidae of the Ethiopian Region. - Transvaal Museum Memoir No. 18: VI + 238 p, 570 figs.

Robinson, G. S. 1979: Clothes-moths of the Tinea pellionella complex: a revision of the world's species (Lepidoptera: Tineidae). - Bulletin of the British Museum (Natural History) Entomology series 38 (3): 57-128, 103 figs.

Petersen, G. 1961a: Tineiden aus SW-Arabien der Ausbeuten H. Scott und E. B. Britton 1937/38. - Beiträge zur naturkundlichen Forschung in Südwestdeutschland 20 (1): 63-65, 3 figs.

Petersen, G. 1961b: Tineiden aus Riad (ZentralArabien) der Ausbeute von Dr. E. Diehl (Lepidoptera: Tineidae). - Beiträge zur naturkundlichen Forschung in Südwestdeutschland 20 (2): 107-110, 3 figs.
Petersen, G. 1964: Tineiden aus Karatschi (Pakistan), Fars (SW-Iran) und den Bahrein-Inseln (Lepidoptera: Tineidae). - Beiträge zur naturkundlichen Forschung in Südwestdeutschland 23 (2): 111-122, 11 figs.

Petersen, G. 1988: Revision der Gattung Ateliotum ZELler, 1839 (Lepidoptera: Tineidae). - Beiträge zur Entomologie 38 (2): 311-324, 53 figs.

Petersen, G. \& Gaedike, R. 1982: Insects of Saudi Arabia. Lepidoptera: Tineidae. - Fauna of Saudi Arabia 4: 333-346, 47 figs.

Petersen, G. \& Gaedike, R. 1984: Beitrag zur Kenntnis der Tineidenfauna des Vorderen und Mittleren Orients (Tineidae, Lep.). - Acta faunistica entomologica Musei Nationalis Pragae 17 (202): 185-212, 30 figs.

Walsingham, T. DE G. \& Hampson, G. F. 1896: On Moths collected at Aden and in Somaliland. - Proceedings of the zoological Society of London 1896: 257-283, pl. X (Figs 1-12, 19-31). 


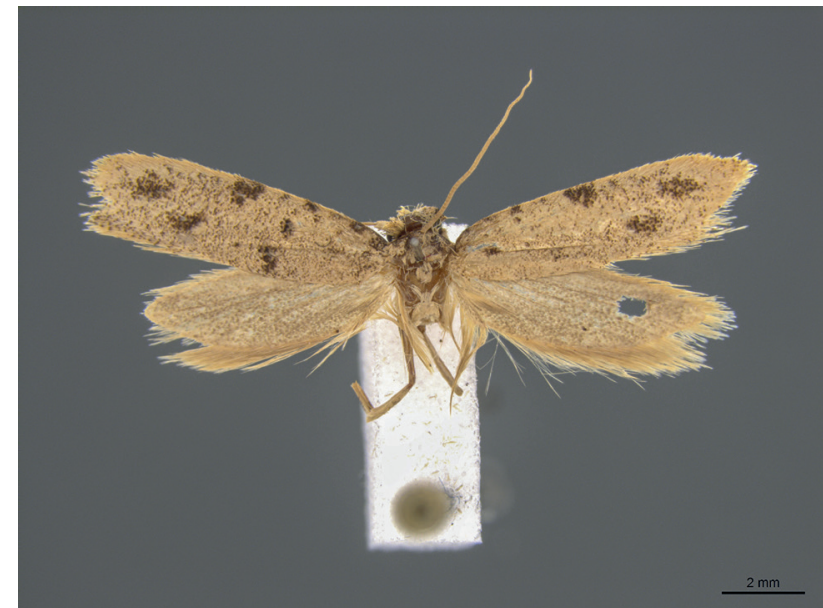

Fig. 1: Hapsifera gozmanyi (holotype).

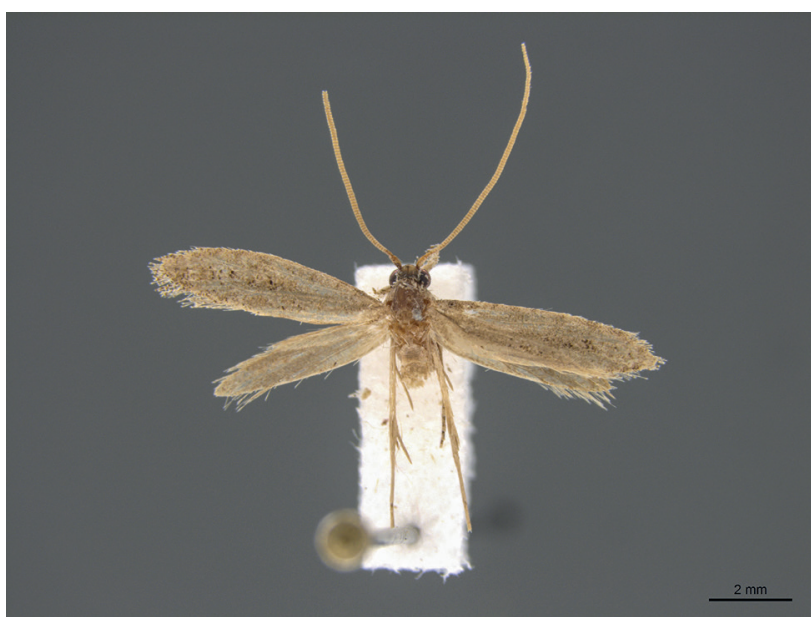

Fig. 3: Hapsiferona jemenitica.

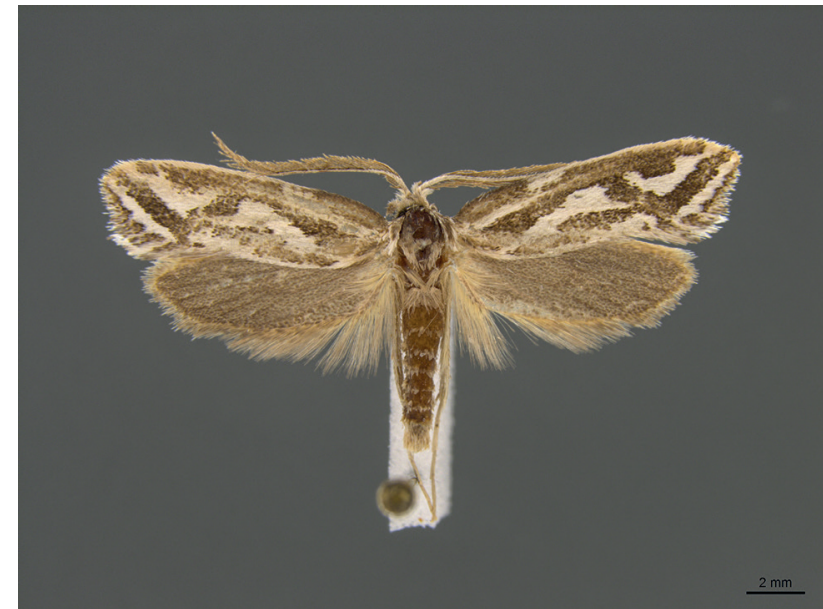

Fig. 5: Psecadioides stuebneri (entire specimen).

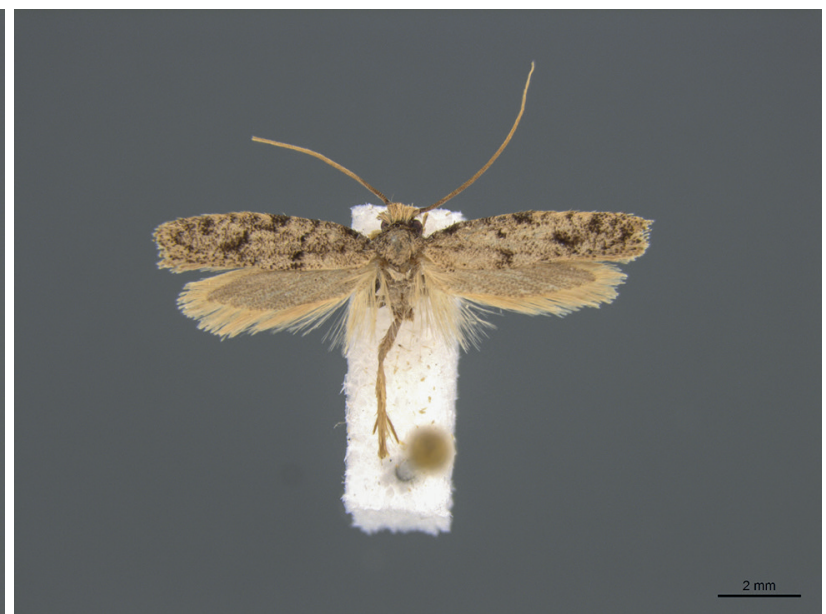

Fig. 2: Hapsifera gozmanyi (paratype).

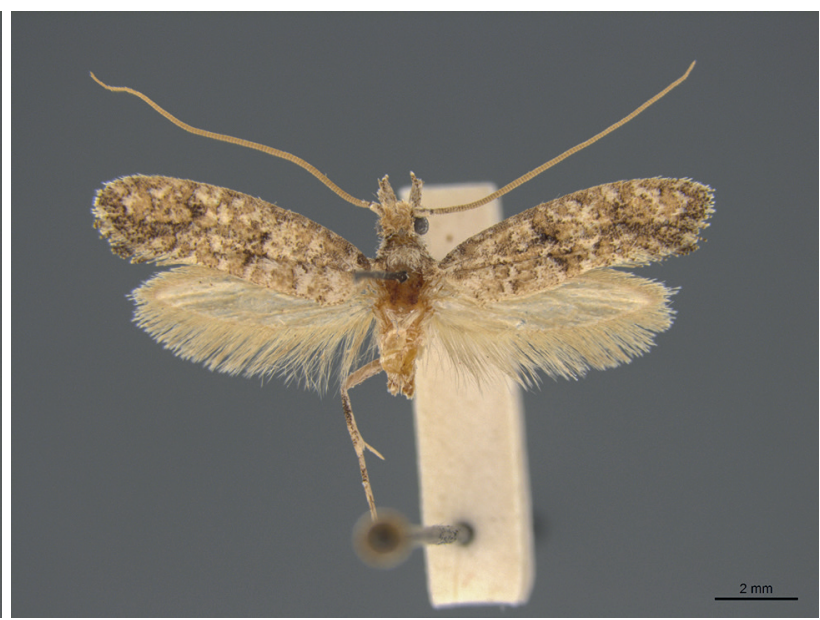

Fig. 4: Hapsiferona remanei.

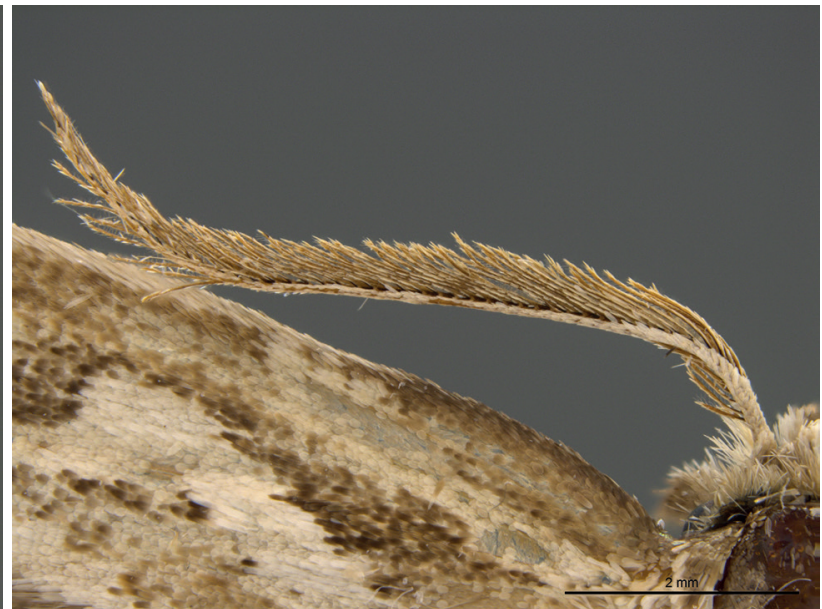

Fig. 6: Psecadioides stuebneri (antenna). 


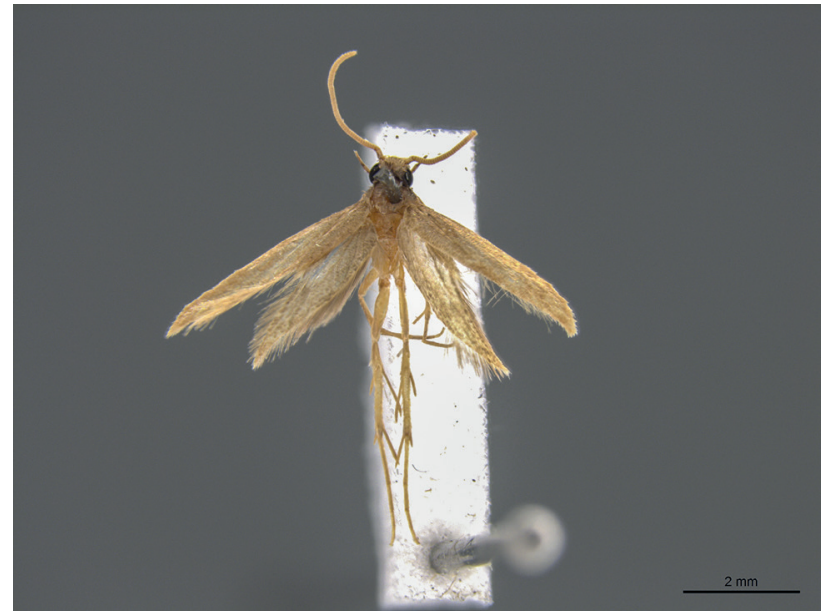

Fig. 7: Heterostasis hackeri.

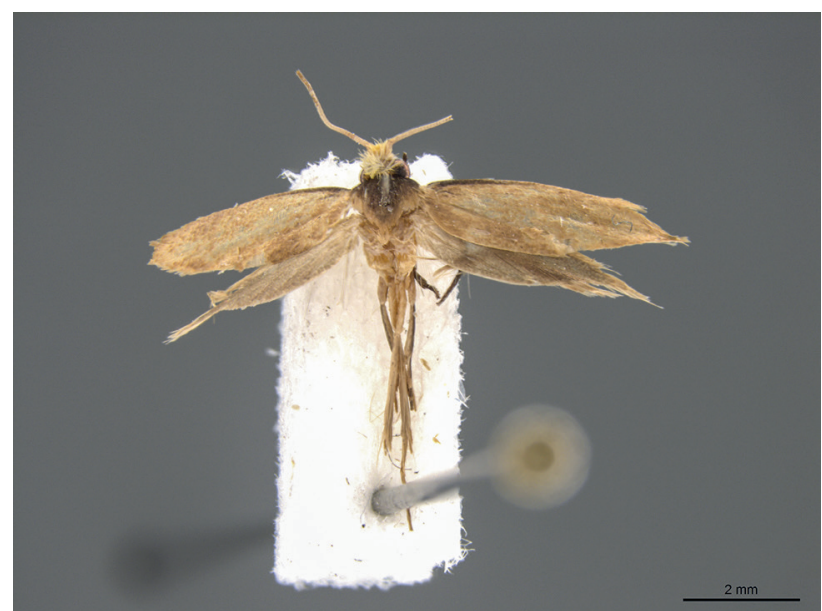

Fig. 9: Edosa pareffulgens.

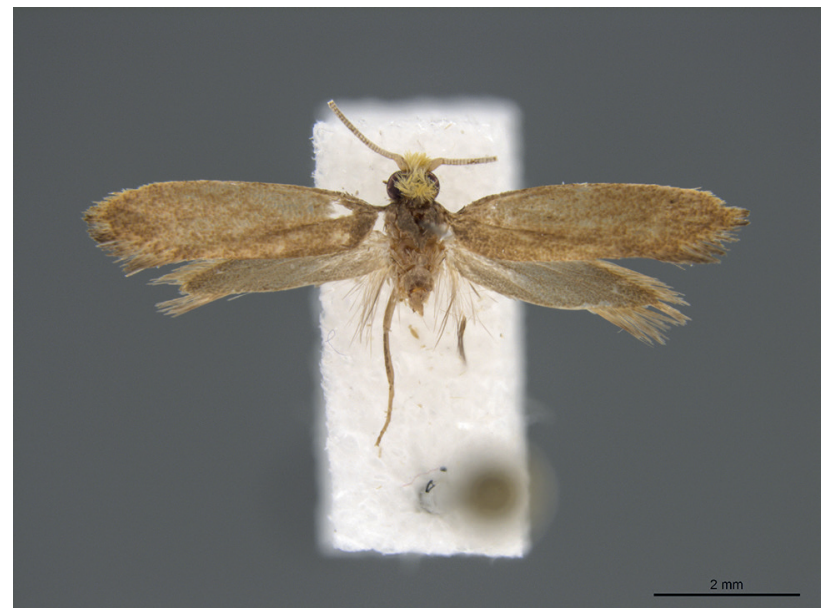

Fig. 11: Edosa furcata.

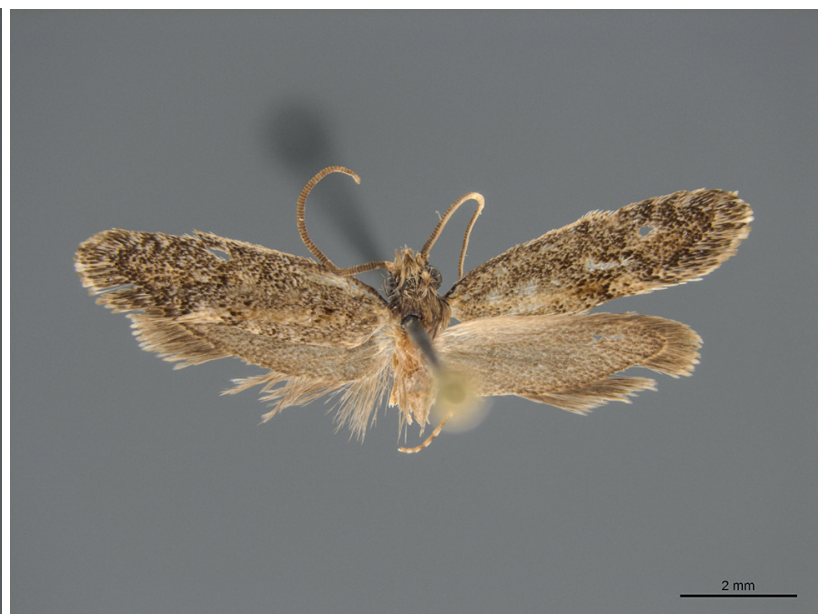

Fig. 8: Pachyarthra africana.

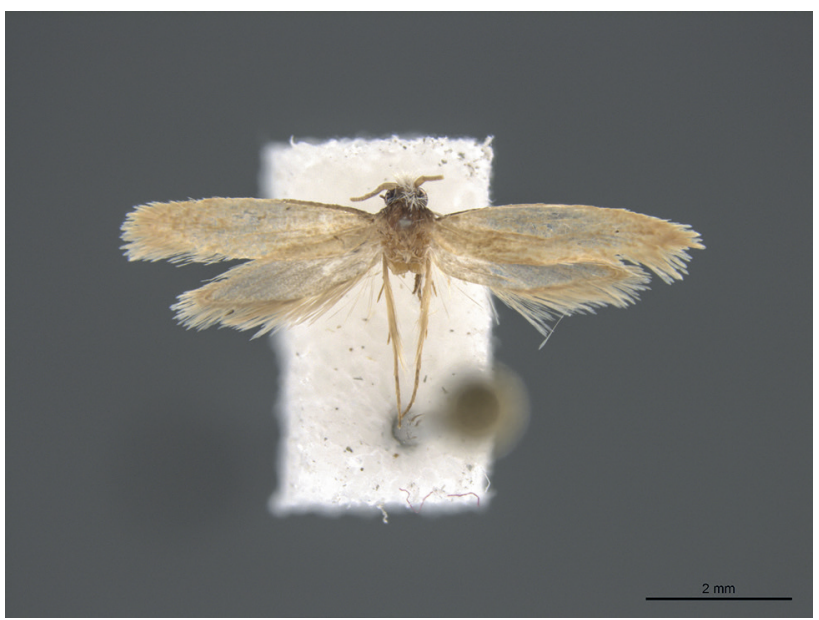

Fig. 10: Edosa albicapitella.

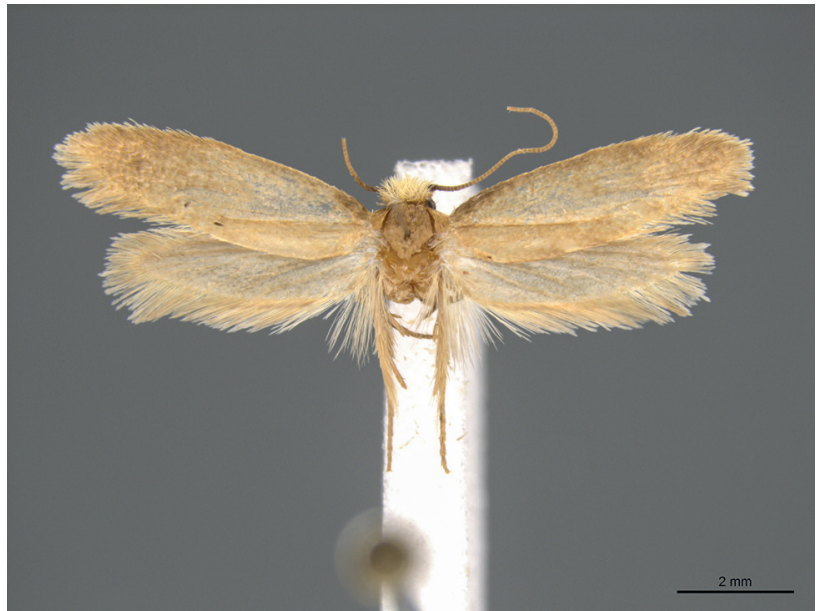

Fig. 12: Hyperbola lehmanni. 


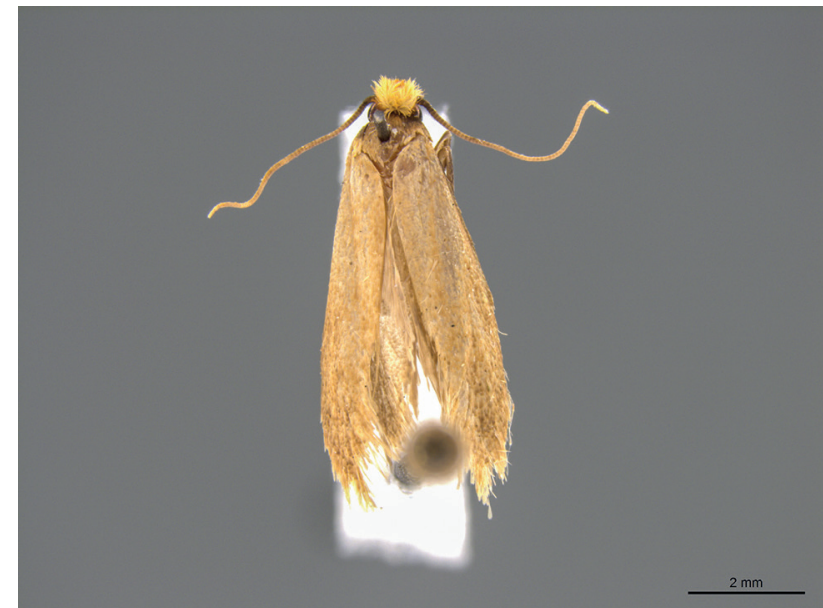

Fig. 13: Hyperbola sexspinosa.

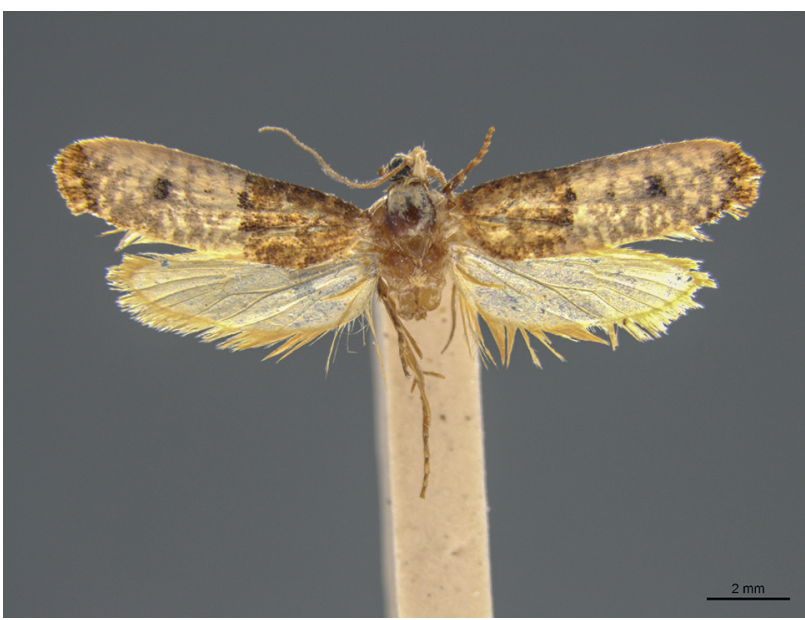

Fig. 15: Trichophaga atripunctella.

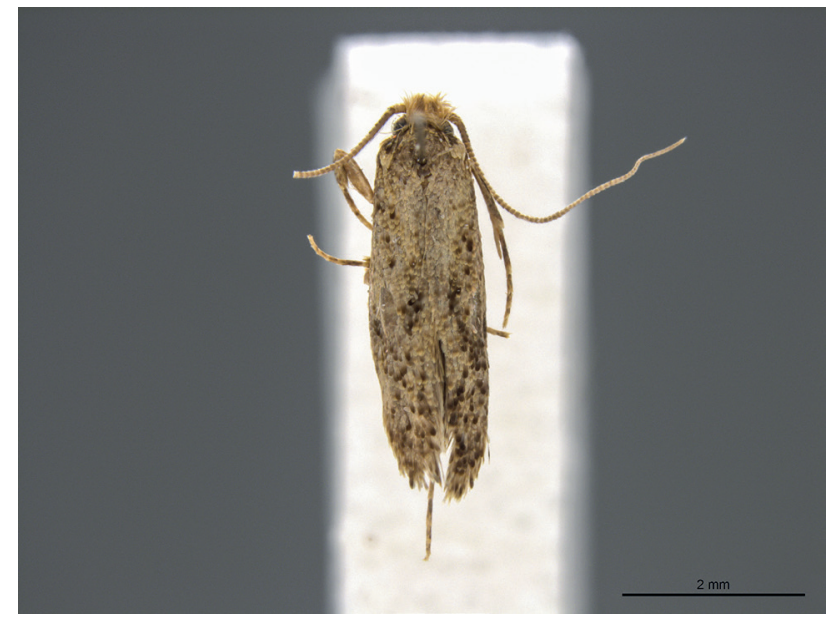

Fig. 17: Proterospastis cornuta.

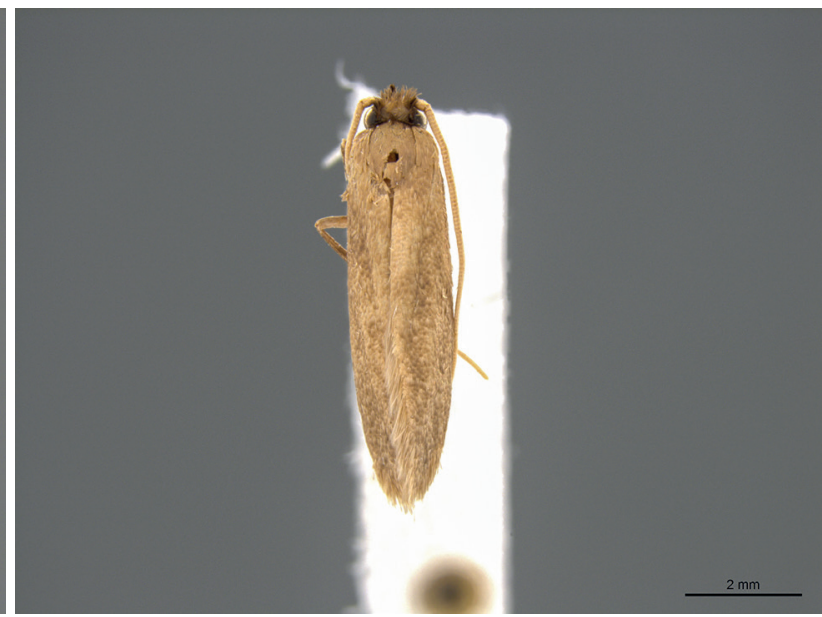

Fig. 14: Perissomastix jemenitica.

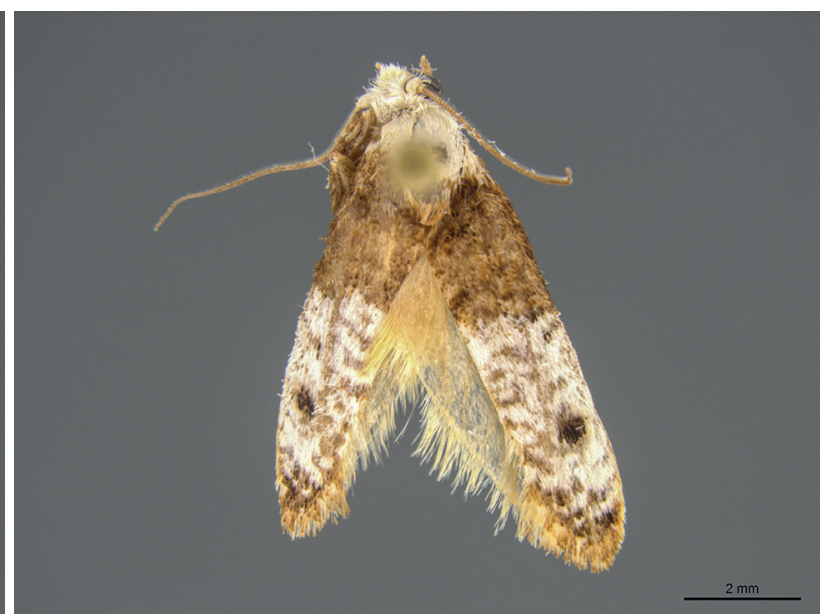

Fig. 16: Trichophaga atripunctella.

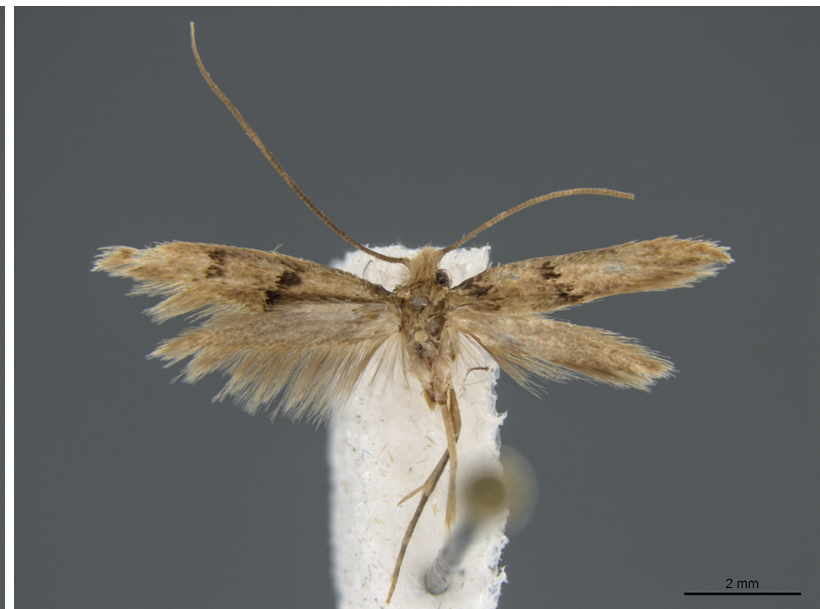

Fig. 18: Proterospastis spinosa. 


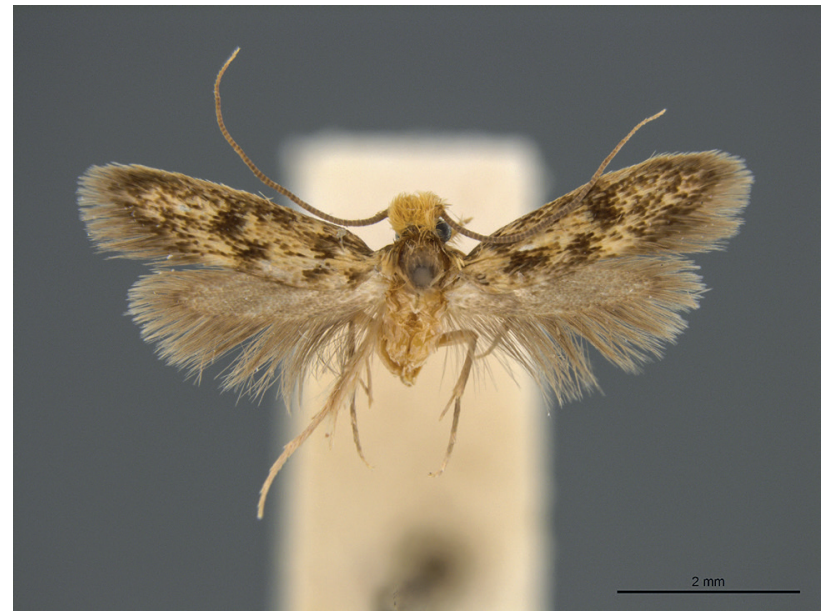

Fig. 19: Crypsithyris luteocapitata.

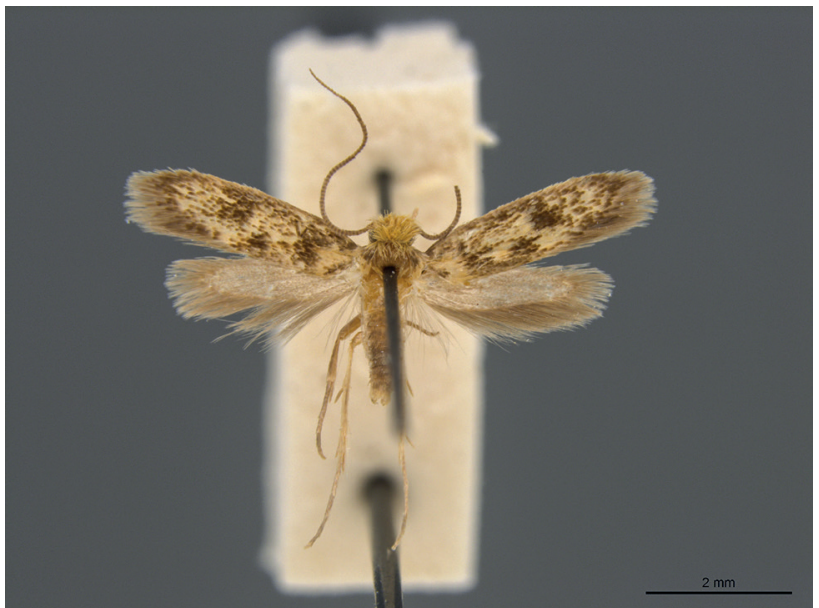

Fig. 20: Crypsithyris luteocapitata.

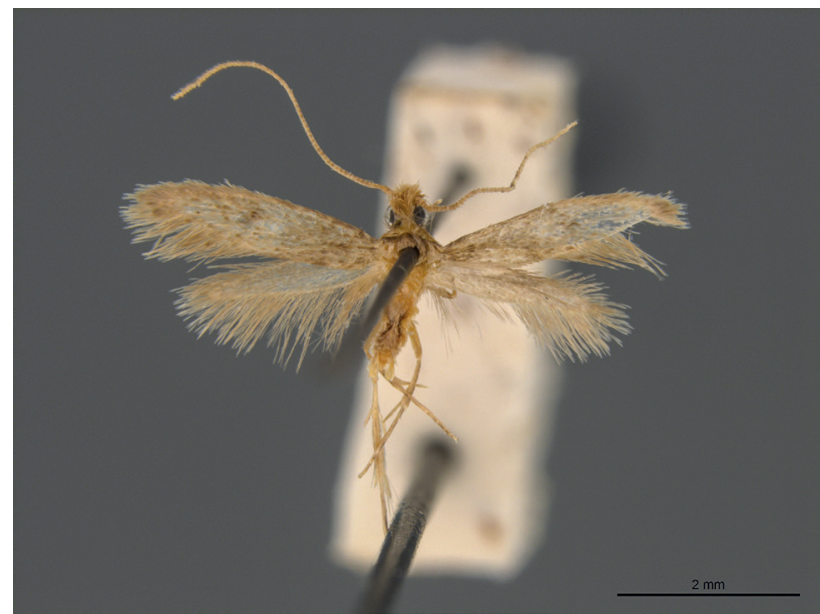

Fig. 21: Crypsithyris unipuncta.

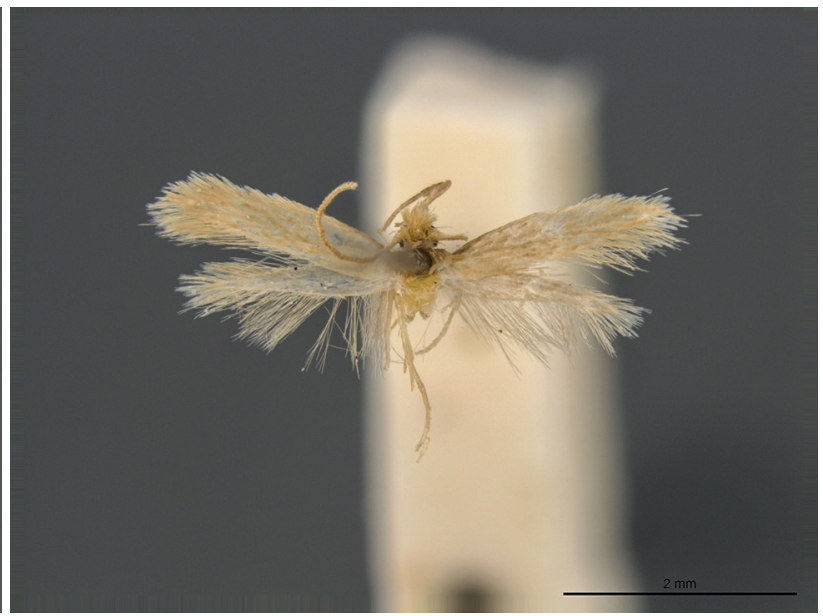

Fig. 22: Ceratobia sudanica.
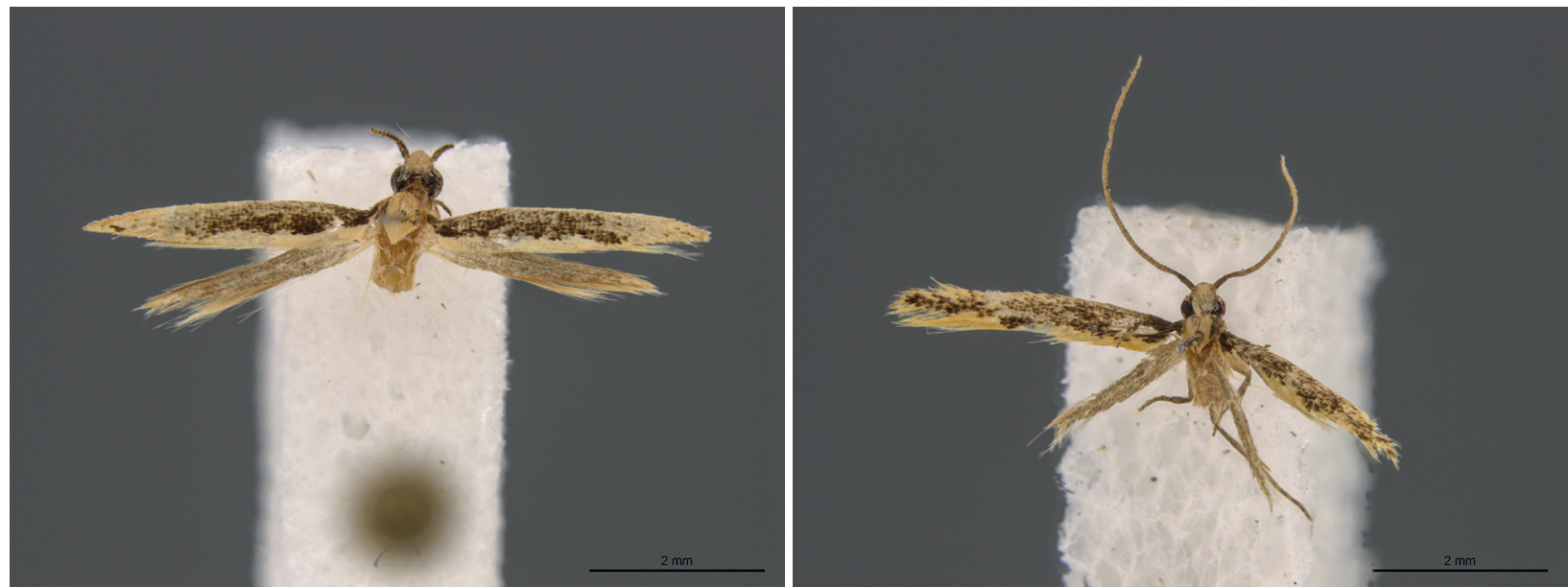

Fig. 23: Amphixystis minuta.

Fig. 24: Amphixystis taurus. 


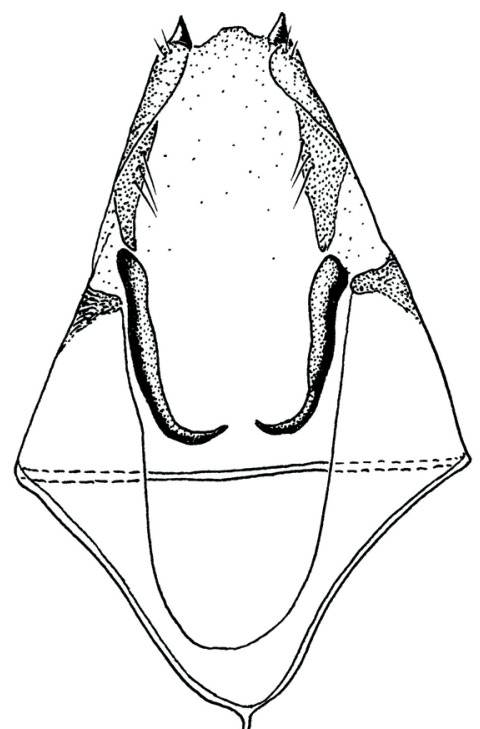

25

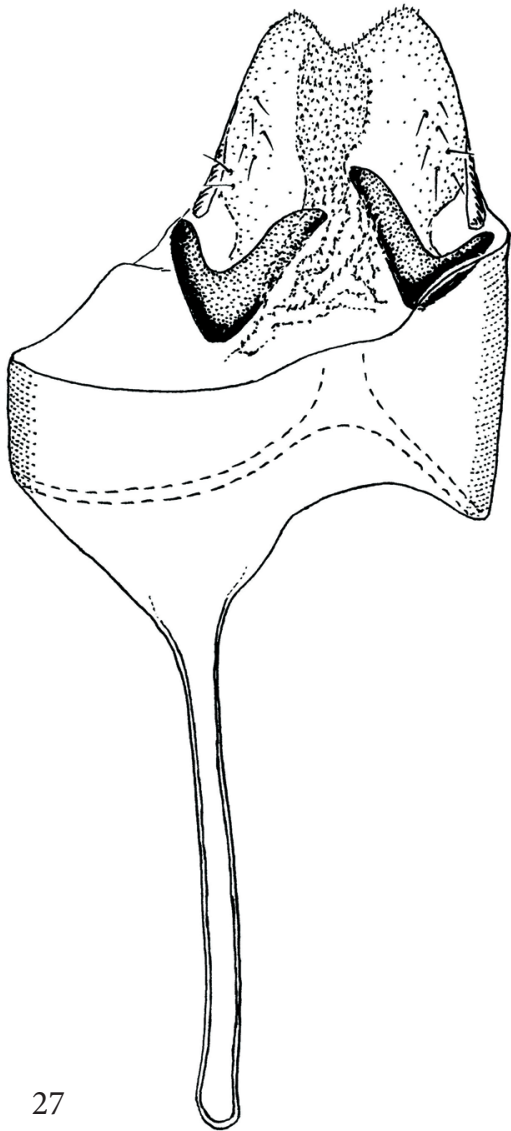

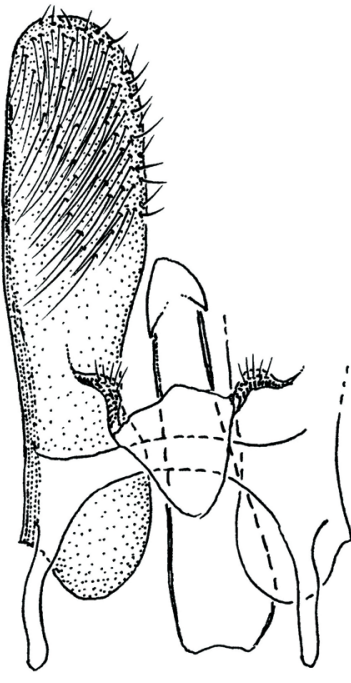

26

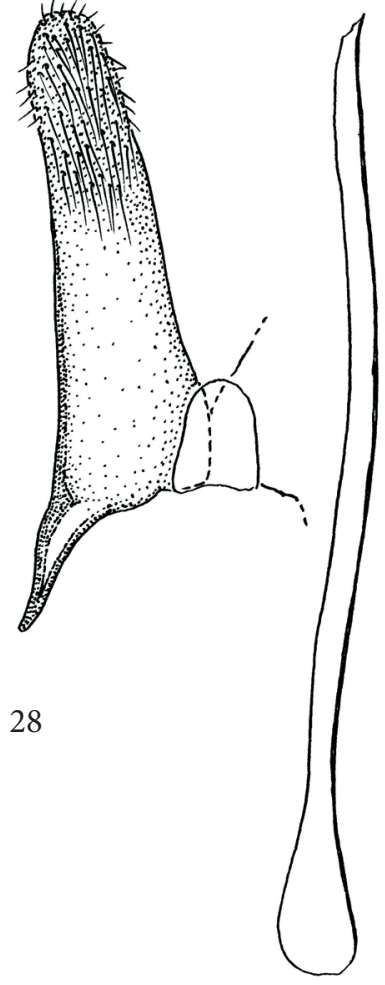

29
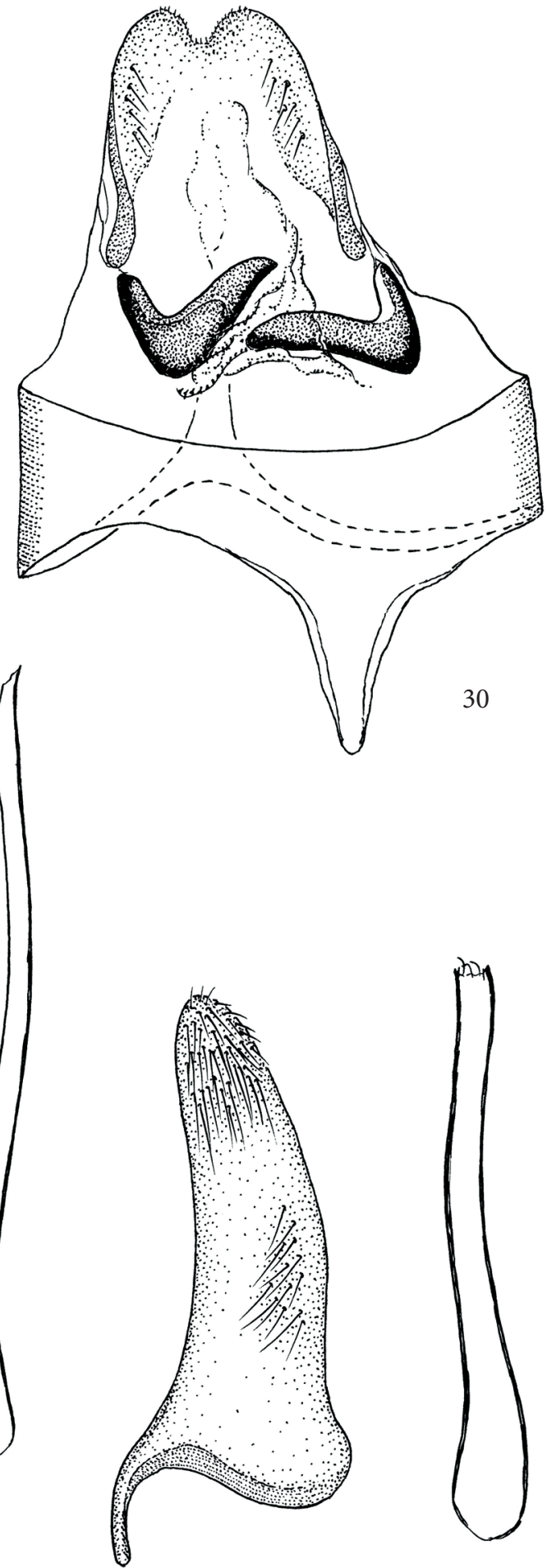

32

Figs 25-32. 25-26: Hapsifera gozmanyi, ơ (25 - Uncus-tegumen complex; 26 - valva and phallus); 27-29: Hapsiferona jemenitica, $\sigma^{\star}$ (27 - Uncus-tegumen complex; 28 - valva; 29 - phallus); 30-32: Hapsiferona remanei, ơ (30 - Uncus-tegumen complex; 31 - valva; 32 - phallus). 


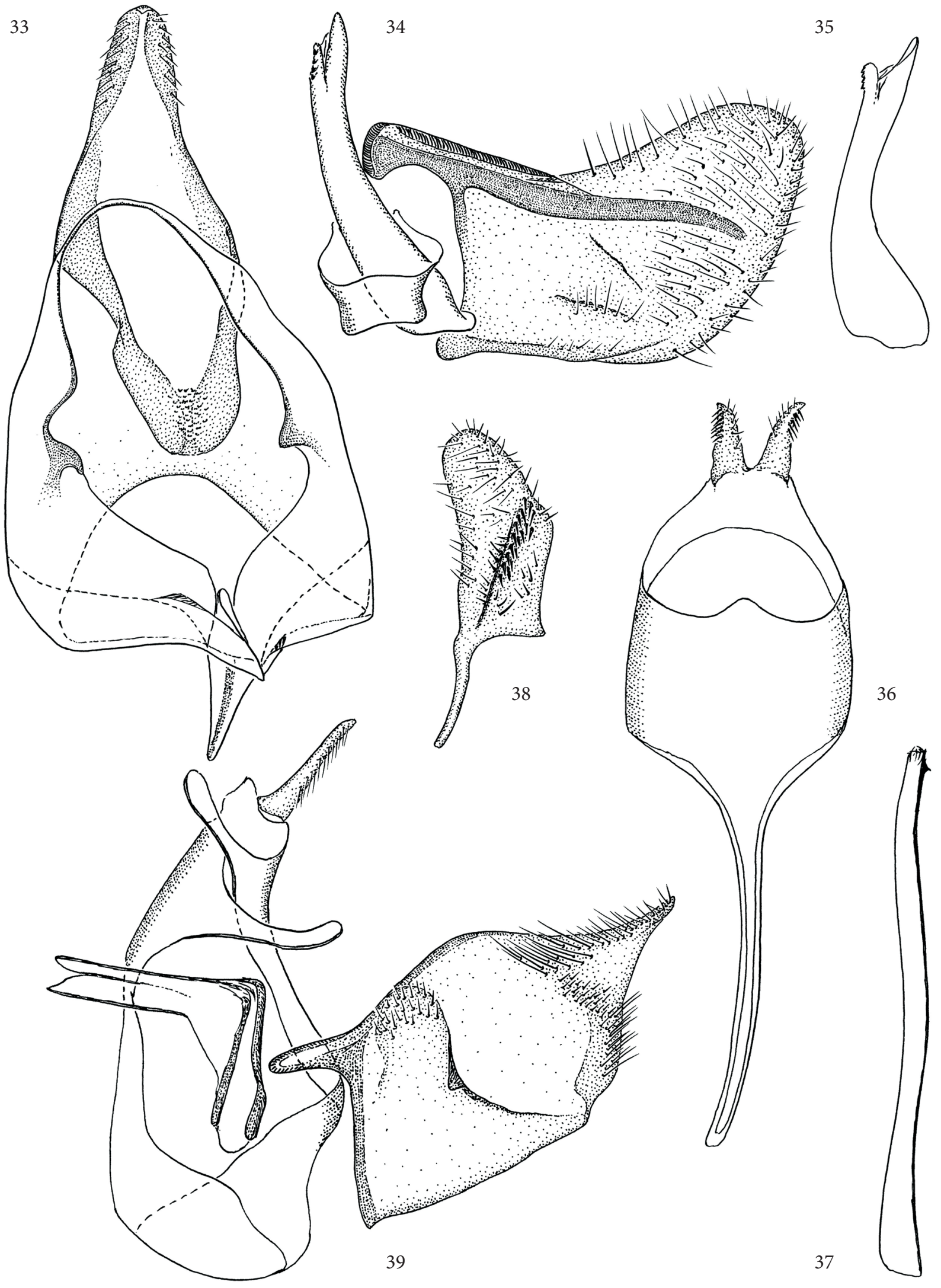

Figs 33-39. 33-35: Psecadioides stuebneri, ơ (33 - Uncus-tegumen complex; 34 - valva, phallus; 35 - other view of phallus); 36-38: Heterostasis hackeri, ơ (36 - Uncus-tegumen complex; 37 - phallus; 38 - valva); 39: Pachyarthra africana, ơ. 

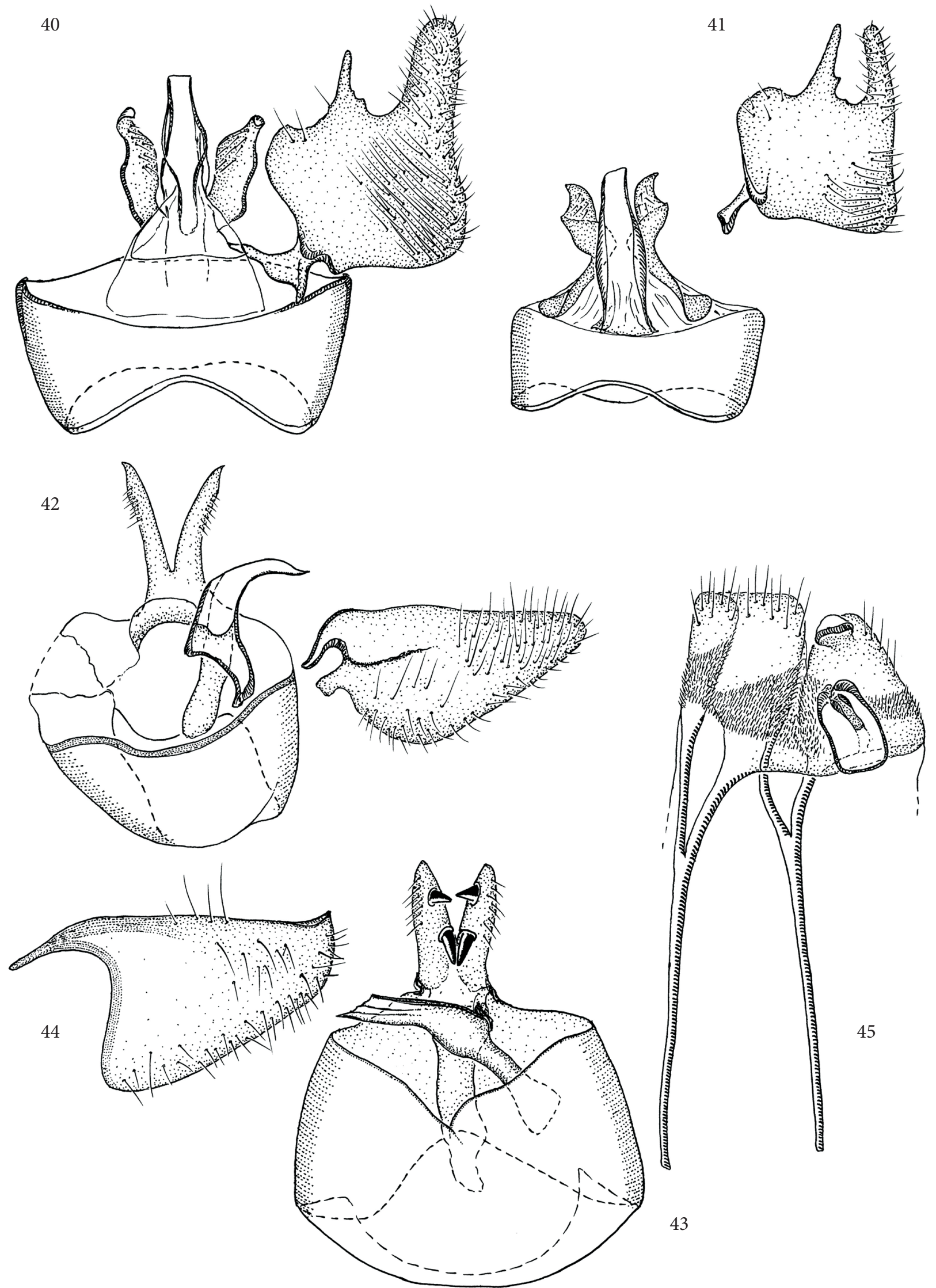

Figs 40-45. 40: Edosa pareffulgens, ơ⿱ ;1: Edosa albicapitella, ơ ; 42: Edosa furcata, ơ ; 43-45: Hyperbola lehmanni, o (43 - Uncustegumen-complex and phallus; 44 - valva); 45: ㅇ․ 

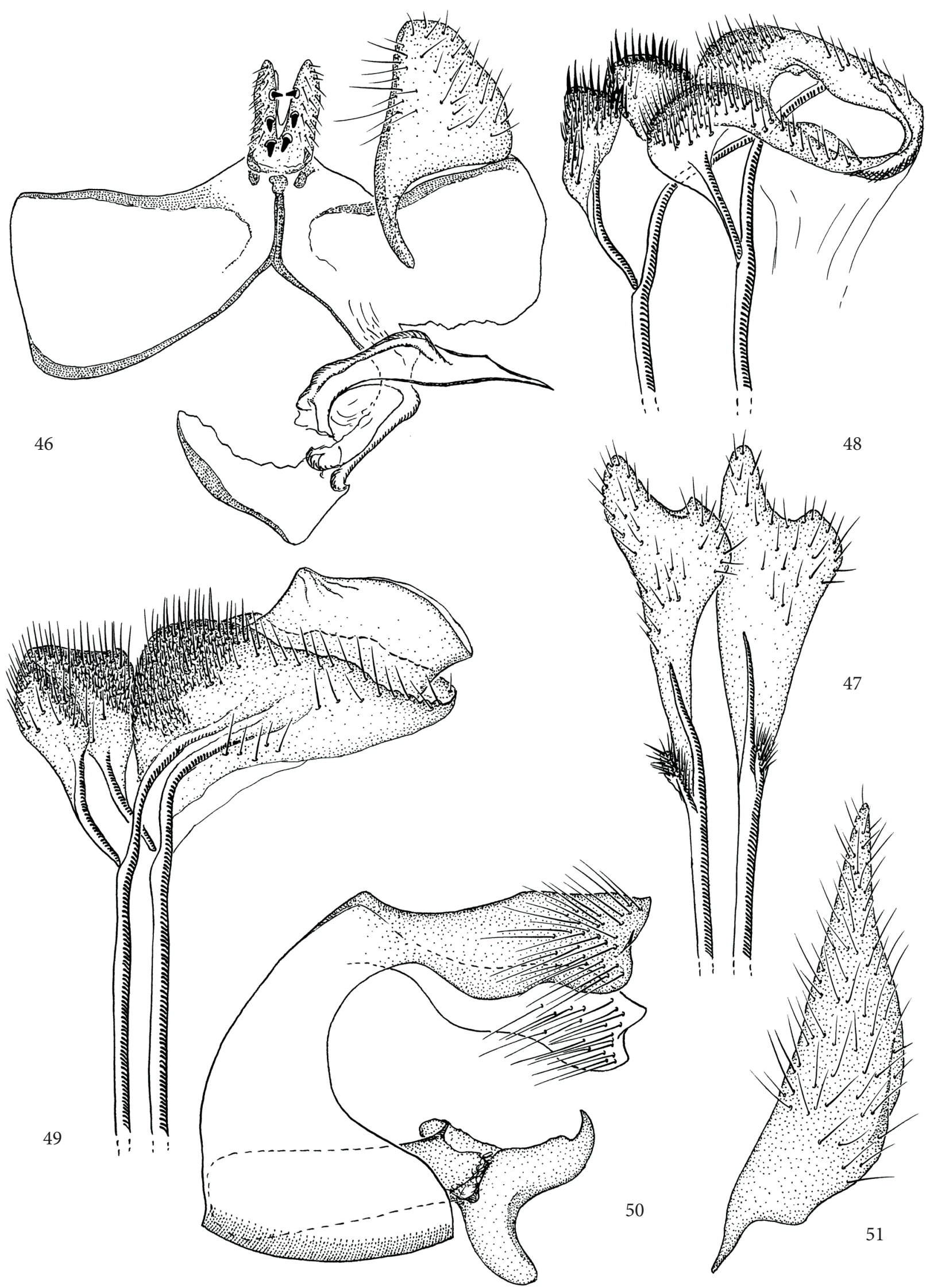

Figs 46-51. 46: Hyperbola sexspinosa, ơ ; 47-48: Perissomastix (Perissomastix) wadimaidaq, o (47 - Papillae anales; 48 - ostium area); 49: Perissomastix (Perissomastix) taeniaecornis,, ; 50-51: Perissomastix (Perissomastix) jemenitica, ơ (50 - Uncus-tegumen-phallus; 51 - valva). 

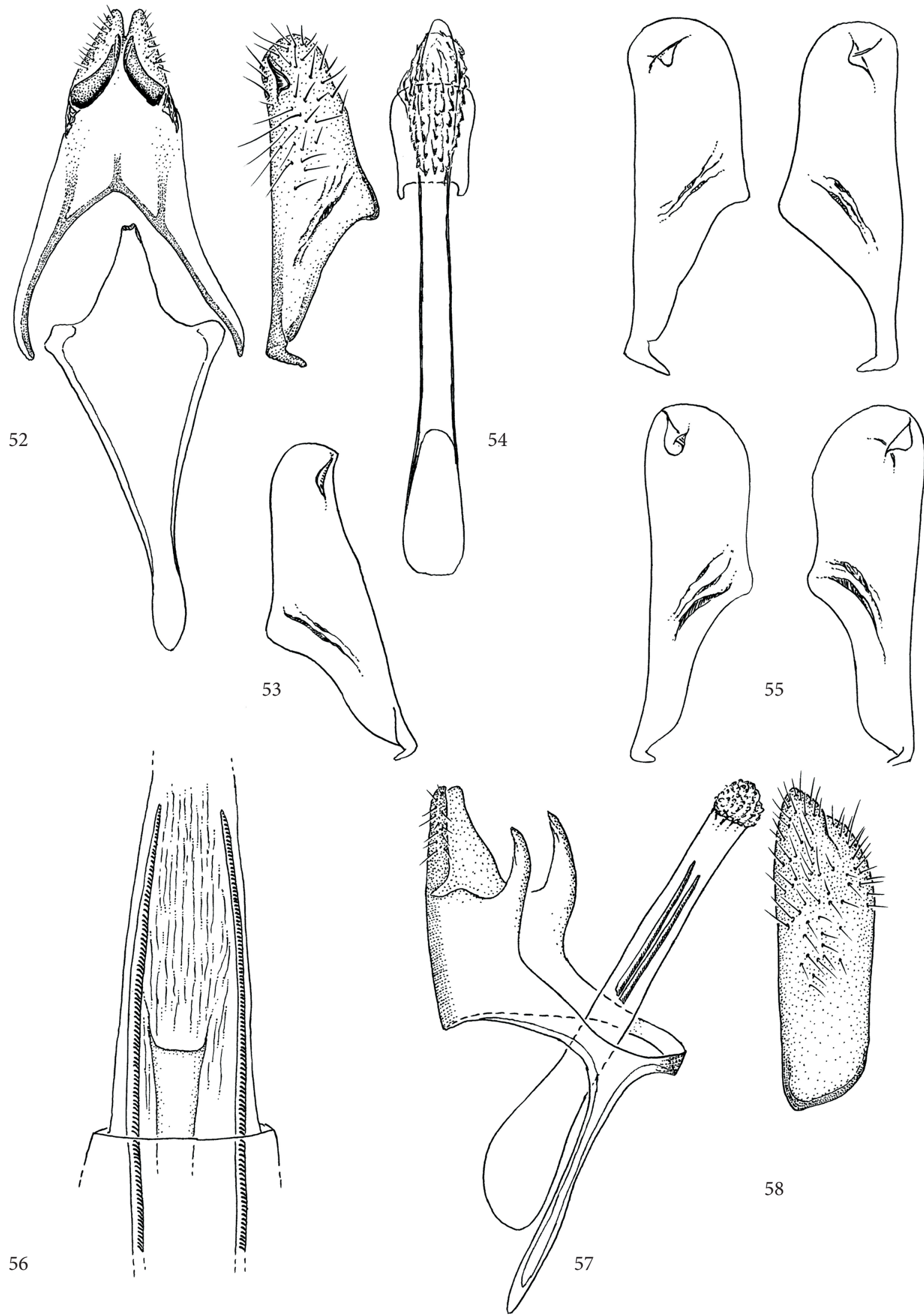

Figs 52-58. 52-56: Trichophaga atripunctella, ơ (52 - Uncus-tegumen-vinculum; 53 - right and left valva; 54 - phallus; 55 - variability of shape of valvae); 56: + ; 57-58: Proterospastis cornuta, ơ (57 - Uncus-tegumen-vinculum, phallus; 58 - valva). 


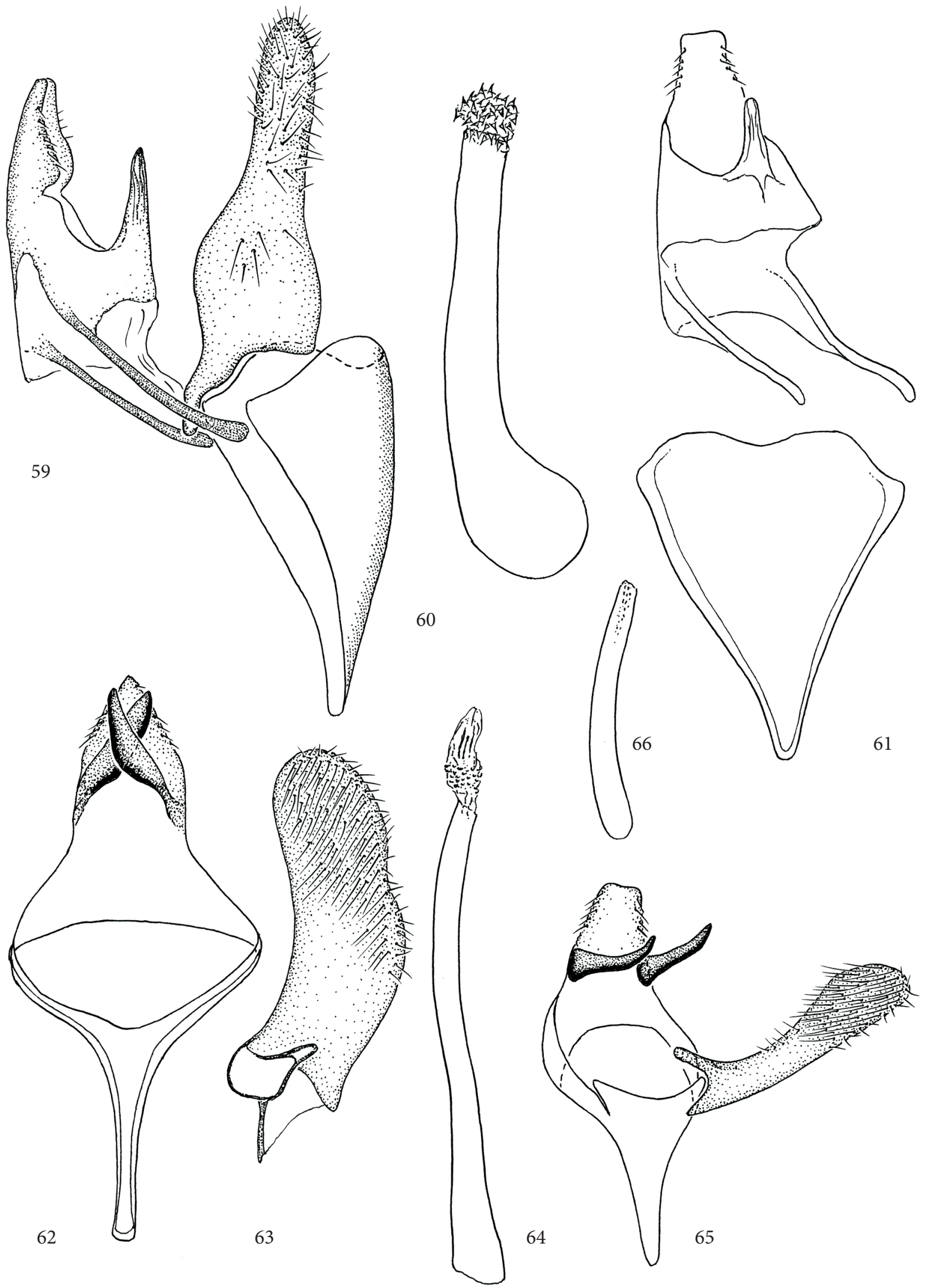

Figs 59-66. 59-61: Proterospastis spinosa, ơ (59 - Uncus-tegumen-vinculum, valva; 60 - phallus; 61 - Uncus-tegumen, and vinculum-saccus, ventro-dorsal view); 62-64: Crypsithyris luteocapitata, ơ (62 - Uncus-tegumen-vinculum; 63 - valva; 64 - phallus); 65-66: Crypsithyris unipuncta, ơ (65 - Uncus-tegumen, valva; 66 - phallus). 


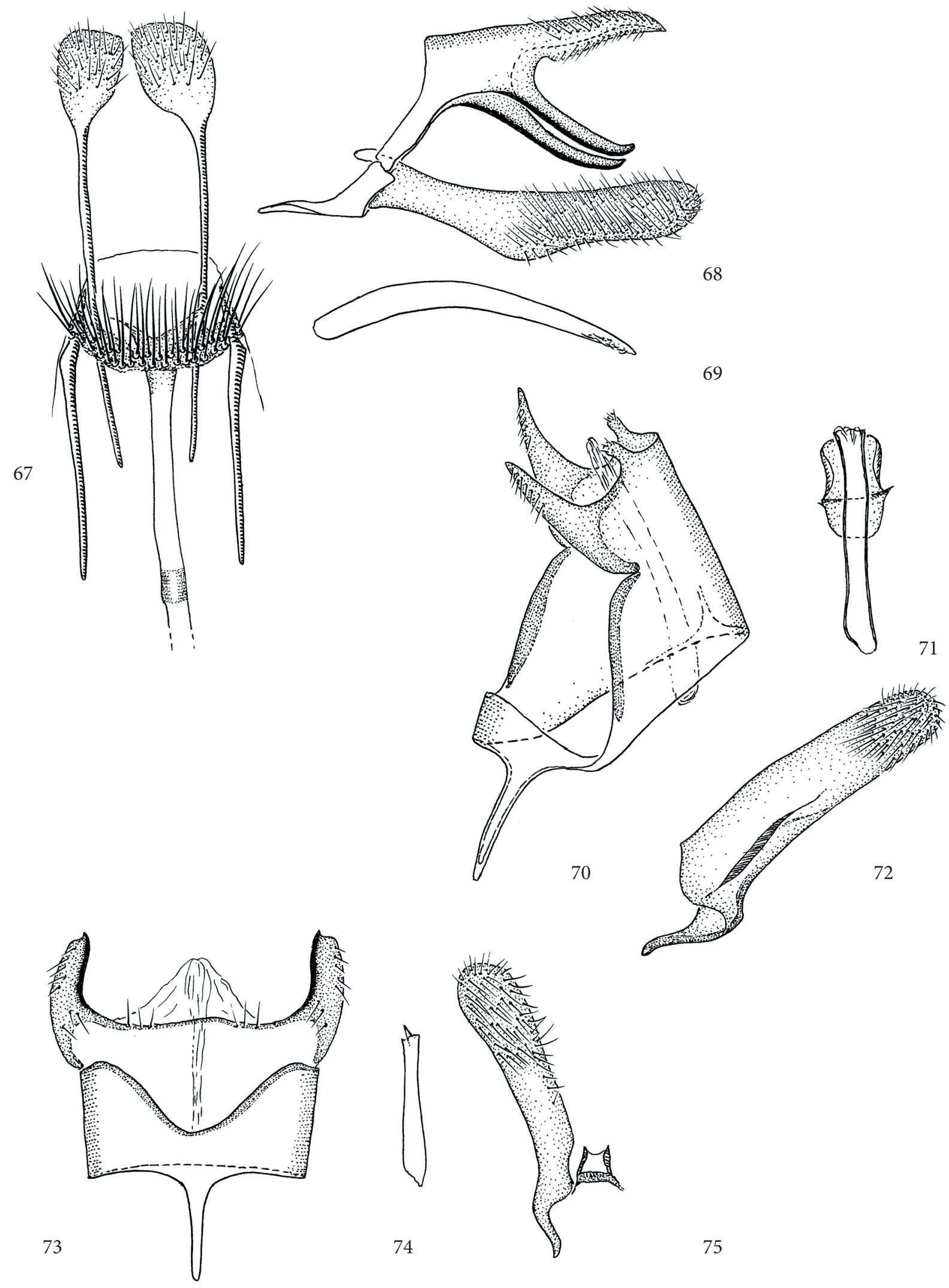

Figs 67-75. 67: Crypsithyris unipuncta, ๆ ; 68-69: Ceratobia sudanica, ơ (68 - Uncus-tegumen, valva; 69 - phallus); 70-71: Amphixystis minuta, ơ (70 - Uncus-tegumen-saccus; 71 - phallus; 72 - valva); 73-75: Amphixystis taurus, ơ (73 - Tegumen-gnathos-vinculum; 74 - phallus; 75 - valva). 